NOTICE: this is the author's version of a work that was accepted for publication in Applied Acoustics. Changes resulting from the publishing process, such as peer review, editing, corrections, structural formatting, and other quality control mechanisms may not be reflected in this document. Changes may have been made to this work since it was submitted for publication. A definitive version was subsequently published in Applied Acoustics, [VOL 99, (December 2015)] DOI 10.1016/ j.apacoust.2015.03.025

\title{
Using spectral finite elements for parametric analysis of the vibration reduction index of heavy junctions oriented to flanking transmissions and EN-12354 prediction method
}

\author{
J. Poblet-Puig*1 and C. Guigou-Carter ${ }^{\dagger 2}$ \\ ${ }^{1}$ Laboratori de Càlcul Numèric, E.T.S. d'Enginyers de Camins, Canals \\ i Ports de Barcelona, Universitat Politècnica de Catalunya \\ ${ }^{2}$ Département Acoustique et Éclairage, Centre Scientifique et \\ Technique du Bâtiment
}

April 13, 2015

\begin{abstract}
The vibration reduction index of heavy junctions is predicted by means of a model based on spectral finite elements. This is equivalent to a finite element method but faster and with smaller computational costs. This advantage is used in order to perform a parametric analysis of the vibration reduction index for several junction types: T-shaped, L-shaped and +-shaped. The influence of several parameters such as: damping, junction dimensions or the mass ratio on the vibration reduction index is observed. The study is focussed to provide data and guidelines oriented to the EN-12354 design method for flanking transmission in buildings.
\end{abstract}

\section{Introduction}

Indirect sound transmissions can be a cause of poor sound insulation. Sometimes the sound insulation provided by single elements is lower than expected due to the flanking transmission paths. Its control is also very important in laboratory set ups in order to make a fair characterisation of the direct airborne insulation of walls [1]. This phenomenon takes place in many situations like vehicles, ships or buildings.

Several models have been developed in order to predict flanking transmissions. Statistical energy analysis (SEA) was used in [2] for the study of long transmission

*correspondence: UPC, Campus Nord B1, Jordi Girona 1, E-08034 Barcelona, Spain, e-mail: jordi.poblet@upc.edu

${ }^{\dagger}$ e-mail: catherine.guigou@cstb.fr 
paths in buildings. A global approach based on a modal description of the structures but supposing weak coupling with fluid zones is proposed in [3]. A more versatile twodimensional vibroacoustic finite element (FEM) model was implemented in [4]. It is able to deal with strongly coupled situations. The vibration transmission through junctions has also been studied with FEM in [5] and [6]. All these methods are complementary in order to cover the whole frequency range considered in building acoustics. Using the FEM is computationally expensive and often limits the frequency range (to low and mid frequencies) or the dimensions of the structures that can be studied. Mainly when dealing with vibroacoustic problems or parametric analyses with large number of situations have to be considered. On the contrary SEA is, in general more adequate at high frequencies with no limitation on computation costs.

A model oriented to building acoustics is developed in [7] and [8]. Its goal is to predict the flanking transmissions in buildings by means of the properties of the isolated components (i.e. walls) and a simple description of their connection. The parameters characterising the individual building components are: the sound reduction index $(R)$, the radiation efficiency $(\sigma)$ and the surface of the partitions $(S)$. The sound reduction index of an indirect path is calculated as

$$
R_{i j}:=10 \log _{10}\left(\frac{1}{\tau_{i j}}\right)=R_{i}+D_{i j}+10 \log _{10}\left(\frac{\sigma_{i}}{\sigma_{j}}\right)+10 \log _{10}\left(\frac{S_{0}}{S_{j}}\right)
$$

where $S_{0}$ is a reference area. $D_{i j}$ is the vibration level difference. It contains the information of the relationship between the components of the building that are in contact (transmission of vibrations between floors, adjacent walls, floors and walls,...) and is defined as

$$
D_{i j}=-10 \log _{10}\left(d_{i j}\right) \text { with } d_{i j}=\frac{<v_{r m s, j}^{2}>}{<v_{r m s, i}^{2}>}
$$

where $v_{r m s, j}$ is the root mean square velocity, $<\bullet>$ means spatial average on the receiving structure $(j)$ or the excited one $(i)$.

In heavy structures, very often it is considered that $\sigma_{i}=\sigma_{j}$ (reasonable for walls with plane faces) or $\sigma_{i} \approx 1$ (due to the low critical frequency) and paths $R_{i j}$ and $R_{j i}$ are averaged. The key parameter in order to evaluate indirect transmissions is the direction averaged vibration level difference $\overline{D_{\nu, i j}}=\left(D_{i j}+D_{j i}\right) / 2$.

This approach is adopted in the standard [9]. In the building, the transmission of vibrations through the junctions of the structure are very important in order to predict the indirect sound insulation. Final design quality highly depends on the uncertainty of input parameters such as $\overline{D_{\nu, i j}}$ [10]. But it depends on the specific situation (dimensions, boundary conditions, damping) or the laboratory conditions. For this reason in the EN-12354 [9] method, the vibration reduction index $K_{i j}$ was defined

$$
K_{i j}=\overline{D_{\nu, i j}}+10 \log _{10}\left(\frac{\ell_{i j}}{\sqrt{a_{i} a_{j}}}\right) \quad \text { with } \quad a_{i}=\frac{2.2 \pi S_{i}}{c T_{i}} \sqrt{\frac{f_{r e f}}{f}}
$$

here $\ell_{i j}$ is the length of the junction, $a_{i}$ is the equivalent absorption length of the wall $i, S_{i}$ its surface, $c$ the speed of sound in the air, $f_{r e f}=1000 \mathrm{~Hz}$ is a reference frequency 
and $T_{i}$ the reverberation time of the wall $i$ that can be calculated as $T_{i}=2.2 /\left(\eta_{\text {total }} f\right)$ (being $\eta_{\text {total }}$ the total loss factor).

$K_{i j}$ is supposed to be invariable or 'situation-independent' (see [11] for detailed explanations). For this reason EN-12354 [9] provides different $K_{i j}$ formulas (Annex E) for different junction types that can be used in each design situation to calculate $\overline{D_{\nu, i j}}$ by means of Eq. (3).

This approach is relatively new [12] and even if it is implemented in the European standard [9], not many experimental data or related models have been reported. The results of laboratory measurements focused on cellular concrete junctions with and without elastic connections were published in [13,14] and in situ measurements on concrete and brick heavy junctions in [15]. Brick-concrete junctions were also tested in the laboratory [16]. The results showed that variation of $K_{i j}$ can be important if there is low modal overlap.

The vibration reduction index $K_{i j}$ has also been determined by means of the FEM in [17] for heavy concrete junctions with and without elastomer to attenuate vibrations. However, there it is commented that computational costs of a three-dimensional FEM model are very high (there is a large number of nodes and unknowns when the mesh is refined due to frequency increase). This limits quite a lot its applicability to low and mid frequencies or to the analysis of few situations. The FEM has also been used in [18] in order to obtain $K_{i j}$ in lightweight junctions. They are more complicated from the geometrical point of view, the variation of materials and the relevance of some construction details like the connection between elements. In theses cases, it seems that formulating a simplified semi-analytical model is much more complicated.

A very related topic is the estimation of the transmission coefficient at junctions (ratio of transmitted and incident power $\gamma=W_{\text {out }} / W_{\text {in }}$ ). It is used in order to study how vibration energy flows in each junction type. Its main application is to derive coupling loss factors required in Statistical Energy Analysis (SEA).

The main difference with the vibration reduction index is the fact that transmission coefficients are usually obtained in a situation where the structures are infinite or semi-infinite and the excitation is an incoming vibration wave (i.e. bending or longitudinal). On the contrary, vibration reduction index $K_{i j}$ is obtained with some excitation acting on the structure (i.e. point forces or acoustic pressure waves) which have finite dimensions. Moreover, transmission coefficients are more related with the energy flow (SEA coupling loss factors) while vibration reduction index is more related with velocity levels or kinetic energy (SEA subsystem energy).

A very well known model in order to estimate the transmission coefficients of junctions is the one presented in [19]. It is formulated for two-dimensional junctions and some hypotheses are required (i.e. equal material properties for all branches) in order to derive the analytical expressions. This is the basis of other improved models in some sense or another. For example [20] studied the asymmetry of junctions, [21] considered elastic elements or hinges used to attenuate the vibration transmission, [22] verified the importance of considering in-plane effects, or [23] analysed the effect of considering finite or semi-infinite branches in the determination of transmission coefficients. Sometimes the transmission coefficients are derived in terms of an average of the incident angle of the vibration wave [24]. Finally, some methodologies to relate the transmission coefficients and the vibration reduction indices have been proposed 
[25] and with the SEA coupling loss factors in [26, 24, 22].

The transmission coefficients and SEA coupling loss factors have also been predicted by means of models considering finite-dimension structures. Modal bases were used in [27] to formulate a methodology that was later verified with experiments in [28]. The FEM was used in [29] to calculate the coupling loss factors through the energies of every coupled element. And a SEA code was compared with three-dimensional FEM in [30].

The goals and contributions of the research presented here are:

- Use of a model based on spectral elements in order to predict the dynamic response of structures in the whole frequency range of interest (up to 5000 $\mathrm{Hz}$ ). It is limited to structures with extrusion symmetry composed of shells (thin plate theory combined with plane stress elasticity, accounting for bending, shear and longitudinal waves). The model is compared with 3D finite elements and published laboratory measurements.

- Provide predictions for the vibration reduction index $K_{i j}$ defined in the EN12354[9]. They are done with a deterministic model that accounts for the real dimensions of the structures (finite and with specific boundary conditions). Mechanical excitation (point forces) are considered.

- Study the vibration transmission behaviour of T, L and +-shaped junctions. The effect of local changes in the thickness is also considered.

The work here is limited to the case of heavy structures due to the range of masses and thicknesses used. Their typology will be uniform which is the case of usual concrete junctions. The prediction of flanking transmissions in lightweight structures is more complicated in the sense that some details play an important role. For example, vibration reduction index must be defined taking into account the wall types [18] (i.e. vibration transmission from a floor to each of the parts of a double wall can be different) or radiation efficiencies can be different depending on the shape in each side of a wall $[31,32]$. Other aspects that are important to adapt current techniques and regulations for lightweight constructions are discussed in [33, 34, 11, 35].

The manuscript is organised as follows. The deterministic model is presented in Section 2, including a comparison with three-dimensional FEM in Section 2.2 and laboratory measurements already published in Section 2.3. The results are shown and analysed in Section 3 before the conclusions.

\section{The deterministic model}

\subsection{General description}

The numerical model is formulated in the frequency-domain and restricted to geometries with extrusion symmetry. It uses spectral finite elements (SFEM), in particular shell strips. The choice of the SFEM is motivated by two reasons. On the one hand, they can perfectly be adapted to this type of geometries with extrusion symmetry (see the T-shaped junction of Fig. 1). The analytical description of variables in the 
extrusion direction $(Y \equiv y)$ is based on trigonometric functions. On the other hand, they are perfectly adapted to dynamic problems and use the solutions of the homogeneous equation as interpolation functions. This is very important in terms of the quality of the solutions because the exact solution is obtained without the need of remeshing. Moreover, the computational costs are drastically reduced. The division of the structure in elements responds only to geometry definition reasons (and not interpolation). It means that, in the T-shaped junction of Fig. 1 only four spectral elements are required: two for the left branch in order to have a node where the force is placed and one element for the other zones (red circles in the figure show the needed nodes).

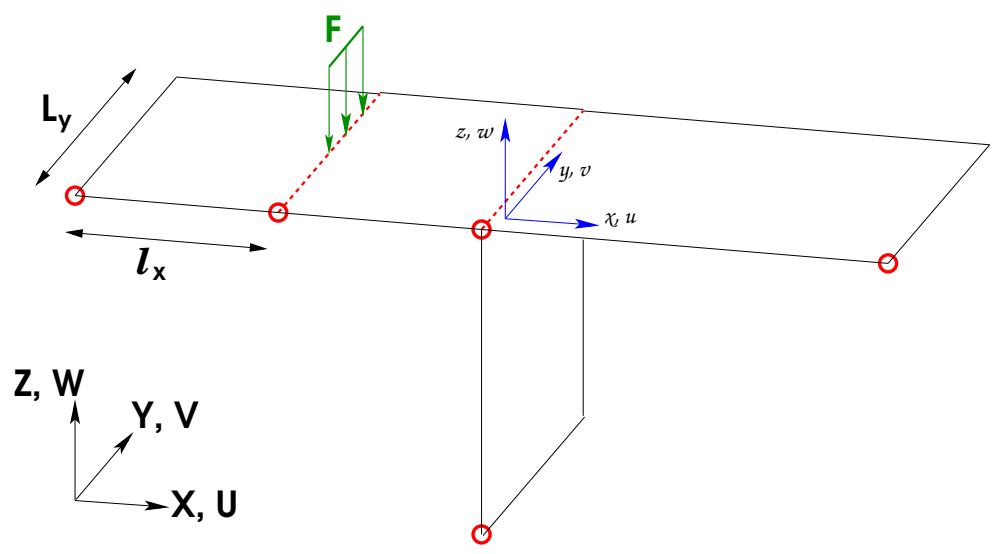

Figure 1: Sketch of the model of a T-shaped junction with four spectral finite elements. Red circles indicate the used nodes (5) for a total of four elements. A narrow strip force is shown (F). $X, Y, Z$ are the global coordinates with displacements $U, V, W$. The length in the extrusion direction is $L_{y}$. The spectral finite element with dimensions $l_{x} \times L_{y}$ has local coordinates $x, y, z$ with local displacemnets $u, v, w$.

To work with a model that allows a deterministic description of the problem in the FEM-style (concise description of the finite dimension geometry with boundary conditions, use of mechanical excitation) but admits fast calculations in the whole frequency range is important in order to reach the goals proposed here. SFEM is a good option to perform a parametric analysis where many three-dimensional problems (frequencies and variation of parameters) must be solved (avoiding the use of big computational resources).

The idea of spectral element (i.e. finite elements for dynamic problems that used fundamental solutions of the equation as interpolation function) has already been exploited in previous works $[36,37]$. However, very often the formulations have small variations in order to adapt to the specific problem studied. For this reason and in order to guarantee reproducibility, the basic details of the in-home implementation are described now. 


\subsubsection{Extrusion direction, harmonics}

The variation along the $Y$ direction is described by means of trigonometric functions (sine series are used here) while a discretisation is done in the $X-Z$ plane. Considering as example, the out-of-plane $(w)$ displacement of an element

$$
w(x, Y, z)=\sum_{n=1}^{N} w_{n}(x, z) \sin \left(\frac{n \pi}{L_{y}} Y\right) \text { with } \xi_{n}=\frac{n \pi}{L_{y}}
$$

where $N$ is the number of terms (harmonics) considered and $L_{y}$ is the problem/element length in $Y$-direction. It means that everything in the $Y$-direction has to be decomposed in contributions according to a Fourier sine series. $w_{n}$ is the description of local displacement at element level in the $X-Z$ plane. Note that due to the extrusion symmetry, the local displacement $w_{n}$ is always parallel to the $X-Z$ plane.

An important advantage of the method is that each contribution $n$ is solved independently from the others. In the remainder of the section the formulation is presented for each contribution ' $n$ ' but the subscript is omitted for clarity (except for parameter $\left.\xi_{n}\right)$. In order to obtain the final result a combination of each uncoupled component ' $n$ ' must be done according to Eq. (4) or its equivalent for the global displacements.

\subsubsection{Elemental matrices of the SFEM shell element}

As usual in thin shell elements, the description of the out-of-plane $(w)$ and in-plane $(u, v)$ displacements is decoupled. The firsts, are related with the bending behaviour and in a spectral element they are interpolated as

$$
w(x, z)=A_{1} e^{-\mathrm{i} k_{1} x}+B_{1} e^{-\mathrm{i} k_{2} x}+C_{1} e^{-\mathrm{i} k_{1}\left(l_{x}-x\right)}+D_{1} e^{-\mathrm{i} k_{2}\left(l_{x}-x\right)}
$$

where $x, z$ are the local coordinates in the element plane, $l_{x}$ is the element length in the $x$ direction.

The key aspect of SFEM is that the interpolation functions are chosen as the solutions of the homogeneous equation. This determines which must be the wavenumbers $k_{1}$ and $k_{2}$

$$
k_{1}=\sqrt{\beta-\xi_{n}^{2}} \quad k_{2}=-\mathrm{i} \sqrt{\beta+\xi_{n}^{2}}
$$

with

$$
\beta=\sqrt{\frac{\rho_{v} t \omega^{2}}{D}} \quad D=\frac{t^{3} E(1+\mathrm{i} \eta)}{12\left(1-\nu^{2}\right)}
$$

Here $\rho_{v}$ is the volumetric density of the shell, $t$ its thickness, $\nu$ the Poisson's ratio, $E$ the Young's modulus, $\omega=2 \pi f, \mathrm{i}=\sqrt{-1}$ the imaginary unit and $\eta$ the hysteretic damping coefficient of the material. The model accounts only for the energy dissipation through the internal damping coefficient of the material. No damping mechanism is considered in the boundaries or the junctions. And the radiation damping is neglected. So, all damping data must be introduced through the internal damping coefficient $\eta$. Afterwards, when applying the correction in order to calculate $K_{i j}$, only the internal damping value used in the model must be considered because it is the energy dissipation that really exists in the numerical model. 
A bending dynamic stiffness matrix for the spectral element can be obtained by means of two steps: i) Express the boundary efforts in terms of the constants $A_{1}$, $B_{1}, C_{1}$ and $D_{1}$; ii) Express the constants $A_{1}, B_{1}, C_{1}$ and $D_{1}$ in terms of the nodal displacements and rotations.

The forces and moments per unit length at the nodes of the element can be expressed as

$$
\begin{aligned}
& F_{z}(x=0, y)=D\left(\left.\frac{d^{3} w}{d x^{3}}\right|_{x=0}-\left.\xi_{n}^{2}(2-\nu) \frac{d w}{d x}\right|_{x=0}\right) \\
& M(x=0, y)=D\left(-\left.\frac{d^{2} w}{d x^{2}}\right|_{x=0}+\left.\xi_{n}^{2} \nu w\right|_{x=0}\right) \\
& F_{z}\left(x=l_{x}, y\right)=D\left(-\left.\frac{d^{3} w}{d x^{3}}\right|_{x=l_{x}}+\left.\xi_{n}^{2}(2-\nu) \frac{d w}{d x}\right|_{x=l_{x}}\right) \\
& M\left(x=l_{x}, y\right)=D\left(\left.\frac{d^{2} w}{d x^{2}}\right|_{x=l_{x}}-\left.\xi_{n}^{2} \nu w\right|_{x=l_{x}}\right)
\end{aligned}
$$

Using the displacement defined in Eq. (5), a relationship of the type

$$
\mathbf{f}_{e}=\left[F_{z}(x=0, y), M(x=0, y), F_{z}\left(x=l_{x}, y\right), M\left(x=l_{x}, y\right)\right]^{t}=\mathbf{B}_{e}\left[A_{1}, B_{1}, C_{1}, D_{1}\right]^{t}
$$

is obtained.

The next step is to express the nodal displacements and rotations in terms of $A_{1}$, $B_{1}, C_{1}$ and $D_{1}$. A $4 \times 4$ system of linear equations is obtained by the evaluation of Eq. (5) at $x=0$ and $x=l_{x}$

$$
\mathbf{u}_{e}=\left[\left.w\right|_{x=0},\left.\frac{d w}{d x}\right|_{x=0},\left.w\right|_{x=l_{x}},\left.\frac{d w}{d x}\right|_{x=l_{x}}\right]^{t}=\mathbf{S}\left[A_{1}, B_{1}, C_{1}, D_{1}\right]^{t}
$$

$\mathbf{S}$ is a small matrix that can be inverted in order to compute the bending dynamic stiffness matrix $\mathbf{K}_{e}^{\text {bending }}$ such that

$$
\mathbf{f}_{e}=\mathbf{K}_{e}^{\text {bending }} \mathbf{u}_{e} \quad \text { with } \mathbf{K}_{e}^{\text {bending }}=\mathbf{B}_{e} \mathbf{S}^{-1}
$$

As shown in [37], in-plane displacements and stresses can be important for high frequencies. Since we deal here with plane elements, the membrane effects are caused by a plane stress state. This is a two-dimensional situation that involves only the in-plane displacements $(u$ and $v)$ and not the out-of-plane $w$. This problem can be solved by means of the Helmholtz potentials (see for example [38]). They are defined here as

$$
\begin{gathered}
\Phi(x, y)=\left(A_{2} e^{-\mathrm{i} k_{P} x}+B_{2} e^{\mathrm{i} k_{P} x}\right) \sin (\xi y) \\
H_{z}(x, y)=\left(C_{2} e^{-\mathrm{i} k_{S V} x}+D_{2} e^{\mathrm{i} k_{S V} x}\right) \cos (\xi y)
\end{gathered}
$$

$\Phi$ and $H_{z}$ must be solutions of the Helmholtz equation with wavenumbers respectively equal to

$$
k_{P}=\sqrt{\frac{\omega^{2} \rho_{v}\left(1-\nu^{2}\right)}{E(1+\mathrm{i} \eta)}-\xi^{2}} \quad k_{S V}=\sqrt{\frac{\omega^{2} \rho_{v}}{G}-\xi^{2}} \quad \text { with } G=\frac{E(1+\mathrm{i} \eta)}{2(1+\nu)}
$$


Once the potentials are defined, all the other variables (displacements, strains, deformations) can be derived according to elasticity equations. The in-plane dynamic stiffness matrix is derived by the same two steps detailed before for the bending part of the shell element. The elemental stiffness matrix is assembled exactly in the same way as for standard FEM.

\subsubsection{Boundary conditions and forces}

All the structures analysed are simply supported at the planes $Y=0$ and $Y=L_{y}$ where the blocked displacements are $U=0$ and $W=0$ ( $V$ is free there). The structures are also supported in the boundary lines that are parallel to the $Y$ direction (three lines in the T-shaped case).

A force in global coordinates can be expressed as

$$
\boldsymbol{F}(X, Y, Z)=\sum_{n=1}^{N} \boldsymbol{f}_{n}(X, Z) \sin \left(\frac{n \pi}{L_{y}} Y\right) \text { with } \xi_{n}=\frac{n \pi}{L_{y}}
$$

and

$$
\boldsymbol{f}_{n}(X, Z)=2 \int_{0}^{L_{y}} \boldsymbol{F}(X, Y, Z) \sin \left(\frac{n \pi}{L_{y}} Y\right) d Y
$$

Considering, as example, the line forces drawn for the horizontal structural element in Fig. 1, that have null component in $Y$ direction and can be expressed as

$$
\boldsymbol{F}(X, Y, Z)=\left(F_{X}=0 ; F_{Y}=0 ; F_{Z}=\left\{\begin{array}{lr}
\delta_{0}(X) & \text { if } Y_{0}<Y<Y_{\text {end }} \\
0 & \text { otherwise }
\end{array}\right)\right.
$$

the $n$ contribution is

$$
\boldsymbol{f}_{n}(X, Z)=\left(0 ; 0 ; \delta_{0}(X) \frac{2}{n \pi}\left(\cos \left(\frac{n \pi Y_{0}}{L_{y}}\right)-\cos \left(\frac{n \pi Y_{\text {end }}}{L_{y}}\right)\right)\right)
$$

each strip force requires to have a node in the position $X_{0}$.

Point forces must be approximated by narrow strip forces due to the trigonometric description of variables in extrusion direction. However, as it will be shown latter, this is not a drawback for the situations analysed here and this approximation is adequate for our purposes.

\subsection{Comparison with FEM}

A comparison with three-dimensional FEM is done here. A T-shaped junction of 0.1 $m$ thick plates is considered with the mechanical properties of Table 3. An sketch is shown in Fig. 1 (notation of the zones in Fig. 5). The dimensions are $L_{x 1}=3.5 \mathrm{~m}$, $L_{x 3}=3 \mathrm{~m}, L_{z 2}=2.5 \mathrm{~m}, L_{y}=4 \mathrm{~m}$ and all boundaries are supported but without constraining rotations and in-plane stresses. The FEM calculation has been done by means of Code-Aster [39] and using DKT shell elements. Three point forces have been considered in zone 1 according to Fig. 5 at the positions: $\left(0.4 \times L_{x 1} \mathrm{~m}, 1.125\right.$ $\mathrm{m}),\left(0.5 \times L_{x 1} \mathrm{~m}, 2.125 \mathrm{~m}\right)$ and $\left(L_{x 1}-1 \mathrm{~m}, 2.875 \mathrm{~m}\right)$. 
The two approaches only differ in: i) The forces are introduced as nodal point forces in the FEM and as narrow strip forces ( $0.25 \mathrm{~m}$ width), explained above, for SFEM; ii) The spatial average is done with numerical integration in the framework of Code-Aster and with arithmetic average of a cloud of points in SFEM. However, these could only cause some differences for very high frequencies (wave lengths much less than $0.25 \mathrm{~m}$, at $5000 \mathrm{~Hz}$ the bending wave length is $0.35 \mathrm{~m}$ ) or when trying to obtain a very precise comparison of the displacements around the zones where forces are applied. The comparison is done in terms of the spatial averaged modulus of displacement in each of the zones.

The results obtained with SFEM are fully equivalent to the ones obtained with FEM. An example is shown in Fig. 3 for the case with $\eta=5 \%$. It is representative of all situations analysed. Quite coarse FEM meshes have been considered in the plot in order to illustrate how the FEM curves separates from SFEM when frequency increases. Moreover, this separation happens in the order of mean element size $h$ (first for the $h=0.7 \mathrm{~m}$, second for the $h=0.5 \mathrm{~m}$ and so on). The FEM curves converge towards SFEM. SFEM is not prone to large errors when frequency increases. Similar results have been obtained for all the displacements in other parts of the junction and when plates are $0.2 \mathrm{~m}$ thick.

The reduction of computational costs due to the use of SFEM is very important. The key steps to be done in SFEM are:

- Create and assemble elemental matrices for very few elements (only needed in order to describe the geometry). These elemental matrices are square (6 rows) and full. It must be done at every frequency and for every harmonic.

- Solve a small linear system of equations. It must be done at every frequency and for every harmonic.

- Post-process. This implies the combination of the $N$ harmonics in the $Y$ direction and must be done for every frequency.

And for the FEM:

- Create and assemble elemental matrices for a larger number of elements (needed in order to describe the vibration waves). Typically mass and stiffness global matrices are created and reused for every frequency. The global matrices can be reused if the mesh is not modified.

- Combine the mass and stiffness matrices in order to create a the matrix of a large linear system of equations. It must be solved at every frequency.

- Post-process. This does not require extra operations and must be done for every frequency.

The costs of SFEM are constant with frequency, they are the same for the low frequencies or for the high frequencies. The reason is that the elements adapt their interpolation of the vibration field to the problem frequency. Consequently the linear systems to be solved are always of the same size. Only the number of harmonics $N$ must be increased. The post-process can be customised (and it is an important 
aspect to optimise). On the contrary, the FEM costs are frequency dependent. They increase due to the need of mesh refinement.

In this T-shaped junction studied here, the size of the SFEM linear systems of equations is $9 \times 9$ and the number of harmonics considered is $N=40$. This number of harmonics is chosen regarding Fig. 2 so that in the zones outside the rectangular shaped strip force, the force value is almost null. Larger values of $N$ should certainly be used in applications where the exact description of the force shape would be crucial or working with narrower strips (less than $25 \mathrm{~cm}$ ) would be needed.

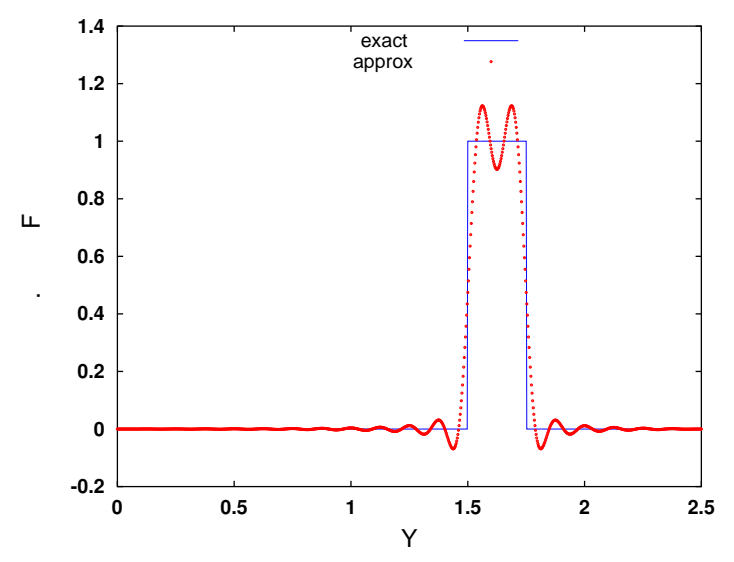

Figure 2: Approximation of a $0.25 \mathrm{~m}$ length line force by means of 40 sines: exact (red), approximation (blue).

In the FEM, for the mesh with mean element size $h=0.3 \mathrm{~m}$, which is not a very fine mesh, the system of linear equations to be solved has around 2000 equations.

The calculation times in this example are $70 \mathrm{~s}$ for SFEM and $15 \mathrm{~s}(h=0.3 \mathrm{~m}), 38$ $\mathrm{s}(h=0.2 \mathrm{~m}), 233 \mathrm{~s}(h=0.1 \mathrm{~m}), 544 \mathrm{~s}(h=0.08 \mathrm{~m})$ for the FEM. This includes 335 frequencies below $1000 \mathrm{~Hz}$. It must be noted that SFEM time is constant while FEM time has an almost cubic grow with the inverse of the element size.

The situation is even more favourable to SFEM if higher frequencies, requiring finer meshes would have been considered. To look at the relative times and the different evolution with frequency is much more important than the final number of seconds. On the one hand, the programs have been run in a simple desktop computer. On the other hand, the SFEM software is a home-made software that could be optimised while Code-Aster is a very big project. The differences are caused by the method. This SFEM model is conceived with a very specific purpose and problem geometries. On the contrary, Code-Aster is a general purpose software.

\subsection{Comparison with experimental data}

The numerical model has been compared with the experimental data published in [13] (Figures 2 and 3). It is the vibration reduction index of a cellular concrete T-junction and + -shaped junction. The geometrical and mechanical properties of the junctions tested are shown in Table 1. Information of the measured damping in the junctions was 


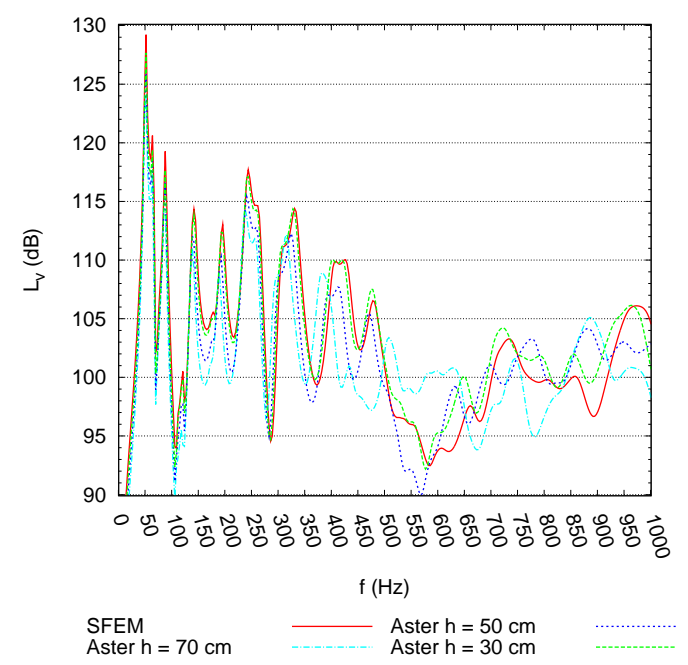

(a)

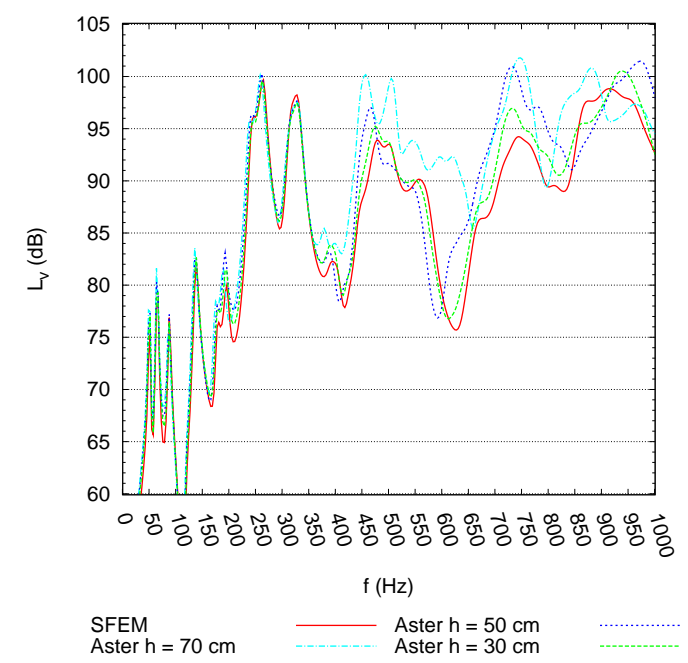

(b)

Figure 3: Comparison between SFEM and FEM (Code-Aster). T-shaped junction, point force excitation in zone 1. Spatial averaged velocity level, $L_{v}=$ $20 \log _{10}\left(<|v|>/ v_{\text {ref }}\right)$, with $v_{\text {ref }}=5 \times 10^{-8} \mathrm{~m} / \mathrm{s}$ : (a) zone 2 , out-of-plane velocity; (b) zone 3, in-plane velocity in the direction $Y$.

provided by the authors [40]. They measured the total damping $\eta_{\text {total }}$ by means of the reverberation time of the structure. It includes the internal damping of the material and the boundary and junction losses. All damping types have been considered by means of material damping in the numerical model. The frequency dependent measure of total damping has been approximated with the values in Table 1.

\begin{tabular}{lll} 
Parameter & Symbol & Value \\
\hline Thickness & $t$ & $0.1 \mathrm{~m}$ \\
Young's modulus & $E$ & $1.61 \cdot 10^{9} \mathrm{~N} / \mathrm{m}^{2}$ \\
Density & $\rho_{s}$ & $624 \mathrm{~kg} / \mathrm{m}^{3}$ \\
Poisson's ratio & $\nu$ & 0.3 \\
Damping & $\eta$ & $3 \%$ \\
& $89-282 \mathrm{~Hz}$ & $2 \%$ \\
& $282-708 \mathrm{~Hz}$ & $2 \%$ \\
& $708-2239 \mathrm{~Hz}$ & $1 \%$ \\
& $2239-5623 \mathrm{~Hz}$ & $0.5 \%$ \\
\hline
\end{tabular}

Table 1: Geometrical and mechanical properties of the plates for the comparison with experimental data [13]

Two different laboratory curves for the vibration reduction index are reported in [13]: transient and stationary. Each of them is based on a different measuring procedure. The experimental data is compared with SFEM predictions in Fig. 4. In the frequency range between $250 \mathrm{~Hz}$ and $1250 \mathrm{~Hz}$, most of the SFEM predicted 
values are between the two experimental curves. In general, the difference between SFEM and laboratory curves is comparable with the average separation between the two measuring procedures. At low frequencies below $200 \mathrm{~Hz}$ differences between the experimental data and the SFEM simulation can be caused by many aspects (i.e. exact type of boundary conditions or force position). At high-frequencies over $2000 \mathrm{~Hz}$ the agreement is not so good. However, the authors [13] mention that 'some research should be carried out' to clarify the increase of the vibration level difference at the higher frequencies. In the SFEM simulations they do not appear. Moreover, the trend seems to go in the inverse sense. Increases of the vibration level difference at high frequencies have only been observed for much larger values of damping. Table 2 shows the arithmetic mean of the vibration reduction index for the third-octave frequency band values between $200 \mathrm{~Hz}$ and $1250 \mathrm{~Hz}$. This frequency range is used in order to obtain the value of $K_{i, j}$ in the EN-12354. The numerical value tends to be closer to the laboratory measurement than the value proposed by the EN-12354. The SFEM value is in general slightly larger than laboratory values, while the value proposed by the EN-12354 is clearly smaller (except for one case).

\begin{tabular}{lrrrr} 
& T-shaped & T-shaped & +-shaped & +-shaped \\
& $K_{13}$ & $K_{12}$ & $K_{13}$ & $K_{12}$ \\
\hline EN-12354 & 5.7 & 5.7 & 8.7 & 8.7 \\
Lab. Transient & 7.1 & 6.5 & 10.0 & 8.5 \\
Lab. Stationary & 7.8 & 7.3 & 10.1 & 8.3 \\
SFEM & 7.5 & 8.2 & 10.8 & 9.7 \\
\hline
\end{tabular}

Table 2: Comparison between EN-12354, laboratory measures [13] and SFEM calculations of the arithmetic mean of the vibration reduction index $K_{i, j}$ for the third-octave values between $200 \mathrm{~Hz}$ and $1250 \mathrm{~Hz}$.

\section{Parametric analysis}

Predictions of the vibration reduction index in ideal L, T, and +-shaped heavy junctions have been done. They are ideal in the sense that the model does not include heterogeneities, boundary losses, cracks or workmanship effects. These can be important in real constructions. Sketches of the junctions are shown in Fig. 5.

The simulations have been done trying to reproduce the laboratory conditions described in the regulation ISO-10848 [41]. Three simultaneous point forces are applied on the zone ' $i$ ' and afterwards three more on the zone ' $j$ ' with the purpose to calculate the direction averaged parameter $\overline{D_{\nu, i j}}$. The forces are placed in arbitrary positions just respecting the limitations described in [41]. The point forces are placed in the same positions mentioned in Section 2.2. They are considered as $25 \mathrm{~cm}$ long line forces. These forces are described by means of $N=40$ contributions according to Eq. (18) (see Fig. 2). The narrow strip forces produce almost the same effect as equivalent point forces. It can be seen in the good agreement with FEM of Section 2.2. Moreover we are more interested in a difference of vibrations between separate parts 


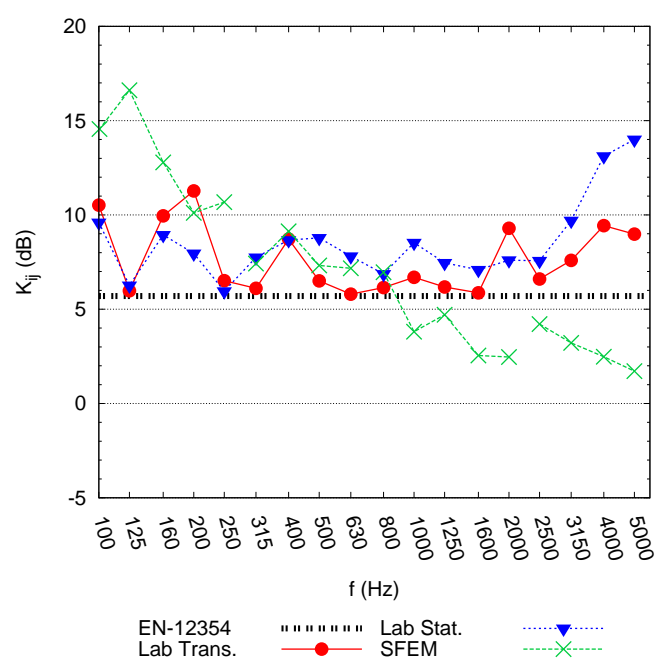

(a)

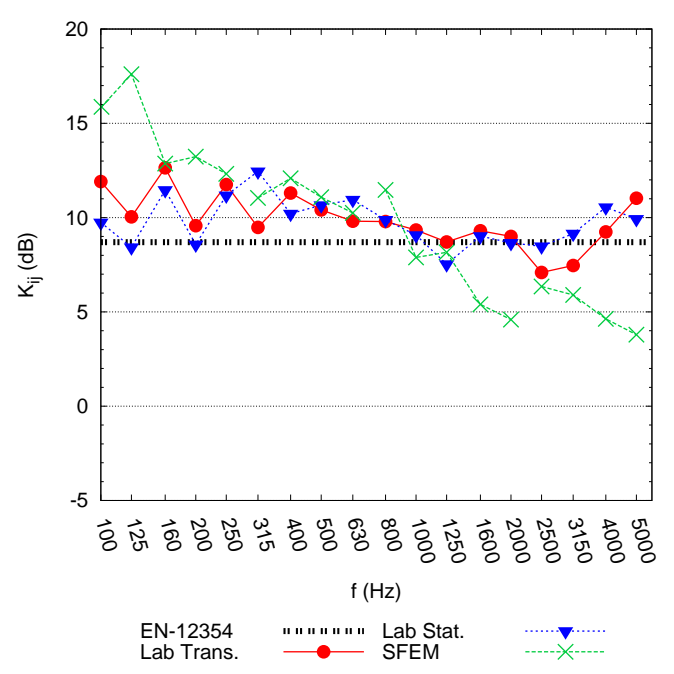

(c)

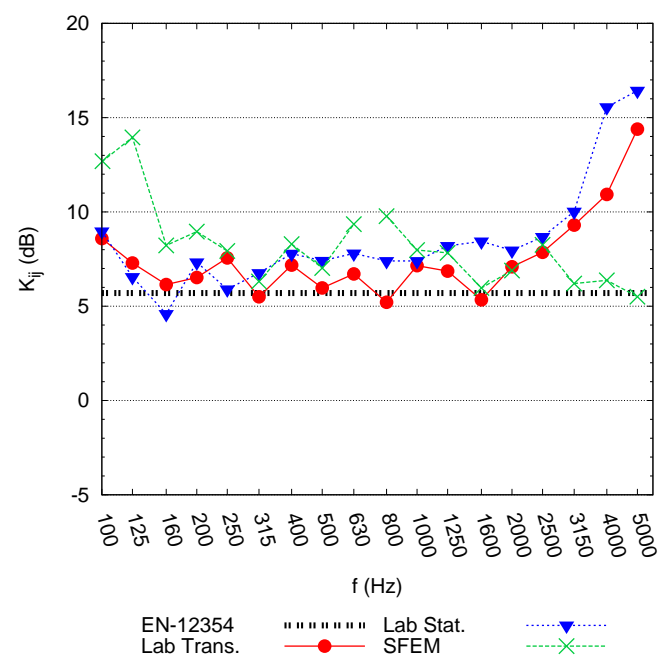

(b)

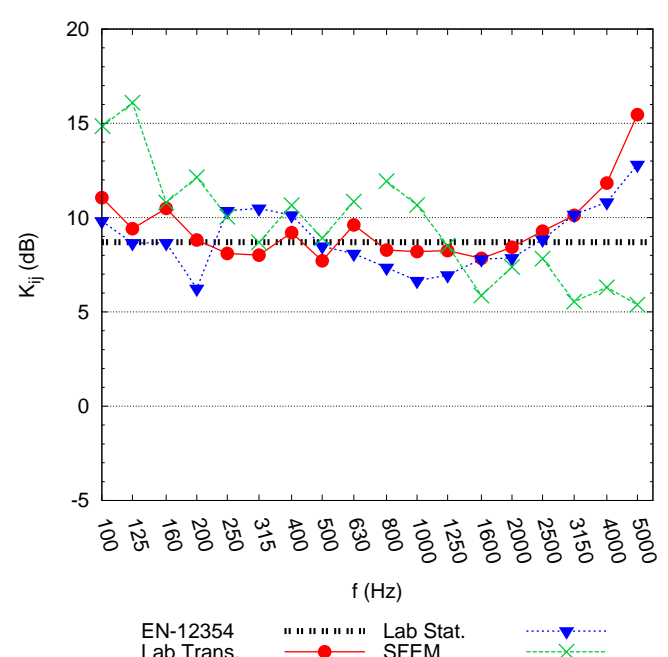

(d)

Figure 4: Vibration reduction index, same case as Fig 2 and 3 in [13]: (a) T-shaped junction, straight transmission $\left(K_{13}\right)$; (b) T-shaped junction, right-angle transmission $\left(K_{12}\right)$; (c) +-shaped junction, straight transmission $\left(K_{13}\right)$; $(\mathrm{d})$ +-shaped junction, right-angle transmission $\left(K_{12}\right)$.

of the structure. Point and narrow strip forces are almost equivalent here (it could be different with airborne excitation).

Calculations have been done in $3 \mathrm{~Hz}$ steps. The results are averaged in thirdoctave bands. The spatial average is performed as the arithmetic mean of discrete values in a uniformly distributed cloud of points (the mean distance between them is $\left.\left(L_{x 1}+L_{x 3}\right) / 20\right)$. Spatial average is done before the frequency average.

When nothing else is mentioned, the mechanical properties of the junctions are the ones in Table 3. The loss factor is a key parameter in the vibration transmission. To study its influence on the response is relevant and a large range of values have 

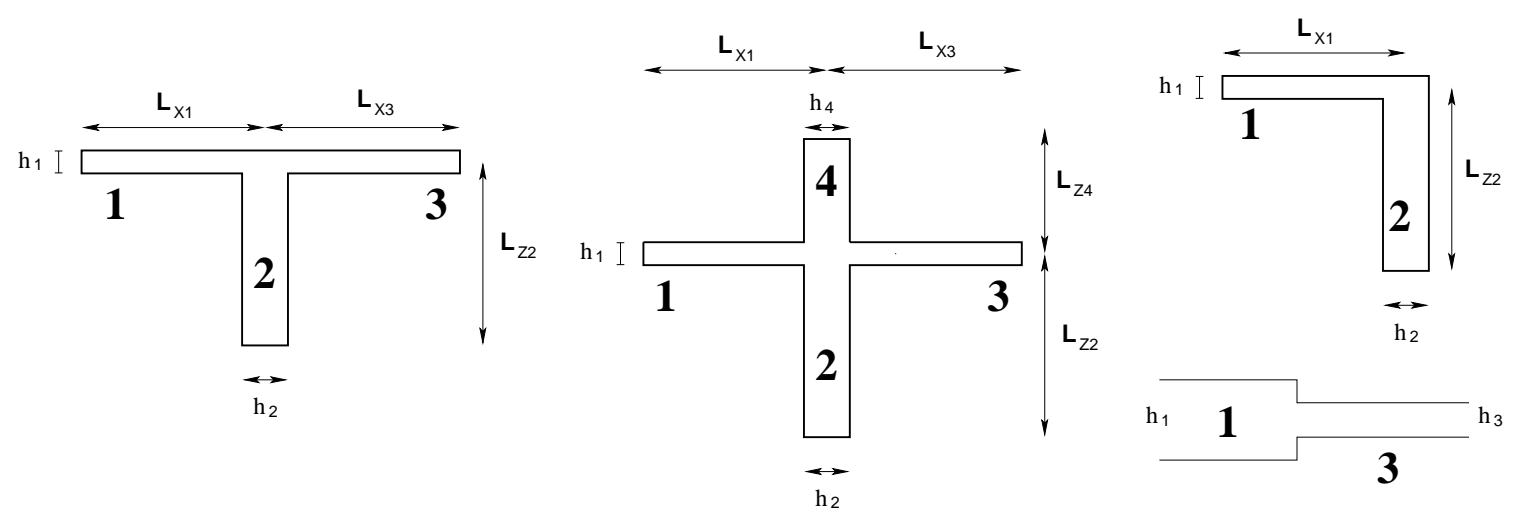

Figure 5: Notation used for the dimensions of the junctions. From left to right: T-shaped, +-shaped, L-shaped and narrowing. $L_{y}$ is the length in the extrusion direction (orthogonal to these cross-sections).

\begin{tabular}{lll} 
Parameter & Symbol & Value \\
\hline Young's modulus & $E$ & $3.0 \cdot 10^{10} \mathrm{~N} / \mathrm{m}^{2}$ \\
Density & $\rho_{v}$ & $2500 \mathrm{~kg} / \mathrm{m}^{3}$ \\
Damping & $\eta$ & $1,5,10,20,30 \%$ \\
Poisson's ratio & $\nu$ & 0.2 \\
\hline
\end{tabular}

Table 3: Mechanical properties of the plates that are constant along the analysis.

been considered. For usual bare construction materials, the internal loss factor $\eta$ is in general below 10\%. However, and as discussed in Section 2, $\eta$ is the only damping parameter considered in the model and all dissipation mechanisms (at boundaries or junctions for example) need to be included there. Consequently, its value is larger than the internal loss factor of the material. Moreover, construction materials with high damping properties are being developed nowadays, see for example the case of polymer concrete [42]. So, a door is open to construct, in the near future, buildings with highly damping structures and junctions.

The aim of Annex E formulas in the EN-12354 [9], resumed in Table 4, is to be a design tool. So, they are quite simple to use and obtained in the basis of large series of in situ measurements in buildings [7, 8] and simplified models for several junctions types [25]. In the remainder of the present work, they are taken as reference.

\begin{tabular}{llll} 
& T-shaped & +-shaped & L-shaped/Narrowing \\
\hline$K_{12}$ & $5.7+5.7 M^{2}$ & $8.7+5.7 M^{2}$ & $\max (15|M|-3,-2)$ \\
$K_{13}$ & $5.7+14.1 M+5.7 M^{2}$ & $8.7+17.1 M+5.7 M^{2}$ & $-5+5 M^{2}$ \\
\hline
\end{tabular}

Table 4: Formulas proposed in Annex E of the EN-12354 [9]. The mass ratio $M=$ $\log _{10}\left(m_{\perp} / m_{i}\right)$ for the vibration transmission path from $i$ to $j, m_{i}$ is the mass per unit surface of element $i$ (i.e. floor) and $m_{\perp}$ is the mass per unit surface of the orthogonal element (i.e. wall). 
In order to keep its purpose of simplicity and universal use, our results are always presented in terms of average of common situations. It means that several combinations of the plates forming the junctions have been considered. The final result is the arithmetic mean of them. The specific dimensions considered can be seen in Table 5 . So, the $K_{i, j}$ results of 12 different T-shaped or L-shaped junctions have been always averaged in order to obtain a more universal analysis, independent of junctions' geometric details. Again, the dimensions are chosen in the line of the recommendations given in [41].

\begin{tabular}{lll} 
Parameter & Symbol & Value \\
\hline Lengths in extrusion direction & $L_{y}$ & $4.0,5.0 \mathrm{~m}$ \\
Lengths for the floors $(\mathrm{T}$ and +$)$ & $L_{x 1}-L_{x 3}$ & $3.5-3.0,4.5-4.0,5.5-5.0 \mathrm{~m}$ \\
Lengths for the floors $(\mathrm{L})$ & $L_{x 1}$ & $3.5,4.5,5.5 \mathrm{~m}$ \\
Lengths for the wall $(\mathrm{T}$ and $\mathrm{L})$ & $L_{z 2}$ & $2.5,3.0 \mathrm{~m}$ \\
Lengths for the wall $(+)$ & $L_{z 2}-L_{z 4}$ & $2.5-2.75,3.0-3.25 \mathrm{~m}$ \\
\hline
\end{tabular}

Table 5: Geometrical properties of the plates.

The calculations are repeated for several combinations of thicknesses in order to have different surface densities and be able to compare with EN-12354 formulas that depend on the mass ratio. In all the junctions analysed, combinations of the following values are considered: $t_{1}, t_{3}=0.1$ or $0.2 \mathrm{~m} ; t_{2}, t_{4}=0.05,0.1,0.2,0.3,0.4$ or $0.5 \mathrm{~m}$.

Several outputs are shown in the following sections:

- Frequency-dependent vibration reduction index curves $K_{i j}(f)$. Results are shown as one-third octave frequency band averages, see for example Figs. 6 and 7. Not less than one value each $3 \mathrm{~Hz}$ is considered in order to perform the one-third octave frequency band average (it makes, for the one-third octave frequency band from $4467 \mathrm{~Hz}$ to $5623 \mathrm{~Hz}$ and central value at $5000 \mathrm{~Hz}, 386$ values). This value is obtained as the arithmetic mean of several situations with different plate dimensions (for example, in the T-shaped junction according to Table 5, there are $3 \times 2 \times 2=12$ situations due to the length variations in,$+ \mathrm{Y}$ and $\mathrm{Z}$ dimensions of the plates).

- Standard deviation of vibration reduction index $\sigma_{K_{i j}}(f)$ due to the geometry of the structure

- Global or 'single-number' vibration reduction index $\overline{K_{i j}}=(1 / n) \sum_{r}^{n} K_{i j}\left(f_{r}\right) \cdot n$ is the number of one-third octave frequency bands considered in the average.

- For the global outputs (or single parameter output like $\overline{K_{i j}}$ ), three frequency ranges have been considered: i)'Low' making the arithmetic average with all one-third octave frequency band results between $50 \mathrm{~Hz}$ and $160 \mathrm{~Hz}$; ii)'Mid' making the arithmetic average with all one-third octave frequency band results between $200 \mathrm{~Hz}$ and $1250 \mathrm{~Hz}$; iii)'High' making the arithmetic average with all one-third octave frequency band results between $1600 \mathrm{~Hz}$ and $5000 \mathrm{~Hz}$. If nothing is mentioned, the default option when global output is shown is the 'Mid' frequency range average. This is the range proposed in [41]. 


\subsection{Influence of damping and structure dimensions}

The study is focused in this section on the influence of geometry variations and the damping. The first aspect is checked by making variations of the plate dimensions and using different thickness ratio of the elements. According to the aim of invariability in the definition of the vibration reduction index $K_{i j}$, very similar results should be obtained for different values of damping and plate dimensions.

\subsubsection{Frequency-dependent curves}

Figs. 6 and 7 illustrated the type of results obtained. The first thing to be mentioned is that the definition of $K_{i j}$ highly reduces the variability of results due to damping. In both figures $\overline{D_{\nu, i j}}(\mathrm{a})$ is compared with $K_{i j}$ (b). While $\overline{D_{\nu, i j}}$ curves present a large range of variation due the different values of loss factor considered, $K_{i j}$ curves are grouped and its differences are in general not larger than $5 \mathrm{~dB}$. This is true except for the curve with $\eta=1 \%$. This damping value is probably too low to fulfil the hypotheses assumed in the definition of $K_{i j}$.

Figs. 6 and 7(a) also show as error-bars, for the cases $\eta=1 \%$ and $\eta=30 \%$, the range of variation of $\overline{D_{\nu, i j}}$ caused by the modification of the plate dimensions (see Table 5). We can observe how it is more important for the smaller values of damping and at low frequencies where the response is controlled by the resonance of single modes.

An important difference with EN-12354 formulas is that here the obtained $K_{i j}$ curves are frequency dependent. And not only in the low-frequency range with the modal behaviour, but also in its global trend. In general, the $K_{i j}$ values predicted by SFEM at low frequencies are higher than EN-12354 estimations mostly due to low modal overlap. This goes in the line of the experimental data published in [25]. They tend to decrease with frequency and remain more or less constant afterwards. At the highest frequencies and for the largest damping values a soft increase of $K_{i j}$ values can be observed. In this frequency range there is enough space in the excited zone to attenuate the vibration waves before arriving to the junction. More excitation force positions have to be considered if a more uniform and reverberant vibration field is required. This is the case of wood junctions where damping tends to be larger [43].

\subsubsection{Statistical approximation of numerical results}

The SFEM results have been approximated by means of a multivariate linear regression analysis. This is useful on the one hand to establish which parameter is more relevant in order to explain the variation of the large amount of data (simulations). On the other hand it is a way to approximate the numerical results without the need of recalculation. It must be mentioned that the cases with $\eta=1 \%$ have been excluded from the statistical analysis due to the large differences observed. The analysis has also been split between low, mid and high-frequency ranges. These decisions are motivated by the different behaviour observed in Figs. 6 and 7 and similar ones. Also the mid-frequency range proposed here is coincident with the ISO 10848-1 [41] in order to calculate the single-number parameter. Thus, the regression formula considered is 


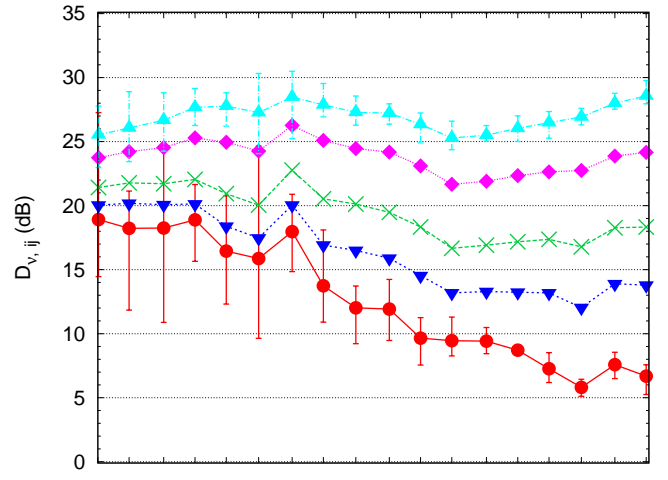

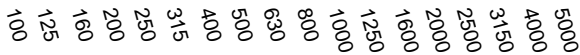

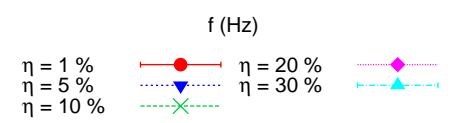

(a)

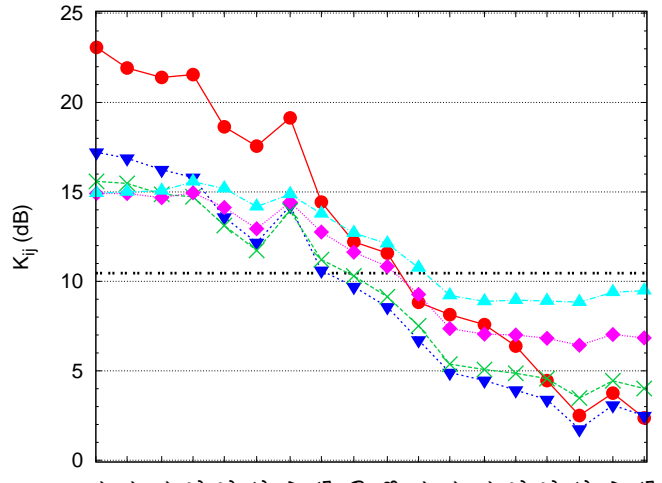

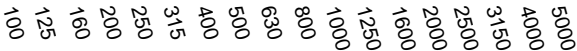

$f(\mathrm{~Hz})$ $\begin{array}{lll}\text { EN-12354 } & \text { SFE........ SFE } \eta=10 \% \\ \text { SFEM } \eta=1 \% & \text { SFEM } \eta=20 \%\end{array}$ $\longrightarrow$ SFEM $\eta=20 \%$

(b)

Figure 6: T-shaped junction, influence of damping $\left(t_{1,3}=0.1 \mathrm{~m}, t_{2}=0.2 \mathrm{~m}\right)$, straight transmission $\left(K_{13}\right)$ : (a) vibration level difference, error bars indicate the maximum and minimum values for all the analysed geometries; (b) vibration reduction index.
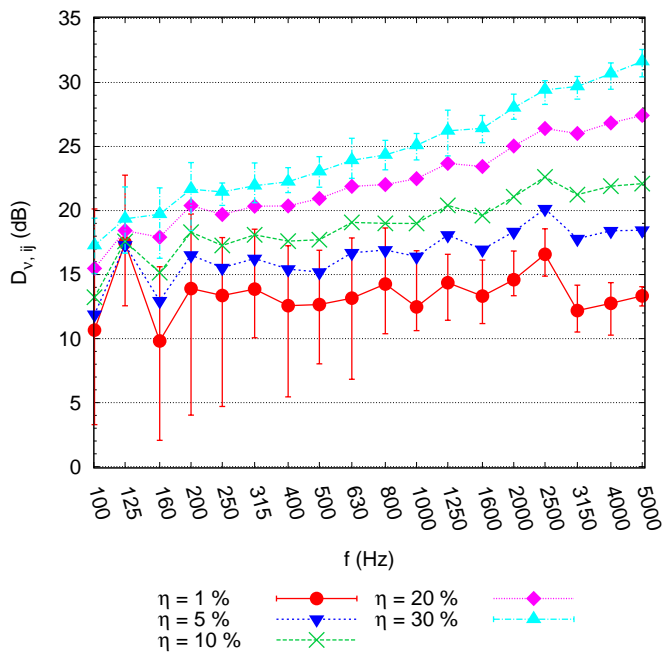

(a)

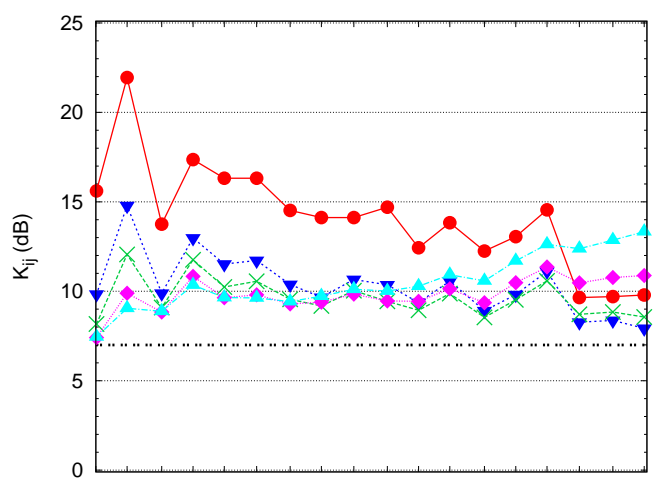

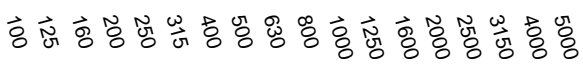

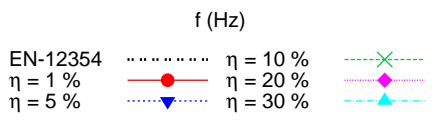

(b)

Figure 7: T-shaped junction, influence of damping $\left(t_{1,3}=0.1 \mathrm{~m}, t_{2}=0.3 \mathrm{~m}\right)$, rightangle transmission $\left(K_{12}\right)$ : (a) vibration level difference,error bars indicate the maximum and minimum values for all the analysed geometries; (b) vibration reduction index. 
as follows

$$
K_{i j}^{\operatorname{Reg}}(f)= \begin{cases}a_{L} \frac{\eta}{100}+b_{L} \log _{10}(f)+c_{L} M+d_{L} M^{2}+e_{L} & \text { if } 44.7 \mathrm{~Hz}<f \leq 178 \mathrm{~Hz} \\ a_{M} \frac{\eta}{100}+b_{M} \log _{10}(f)+c_{M} M+d_{M} M^{2}+e_{M} & \text { if } 178 \mathrm{~Hz}<f \leq 1413 \mathrm{~Hz} \\ a_{H} \frac{\eta}{100}+b_{H} \log _{10}(f)+c_{H} M+d_{H} M^{2}+e_{H} & \text { if } 1413 \mathrm{~Hz}<f \leq 5623 \mathrm{~Hz}\end{cases}
$$

This is based on the EN-12354 formulas but adding the effect of frequency dependence and damping. Both are considered by means of a linear dependence on $\log _{10}(f)$ and $\eta$. The variables $\left(\eta, \log _{10}(f), M \ldots\right)$ are considered or not, depending on its influence on the linear regression analysis. In a first iteration all independent variables are included. The significance of each one is checked which helps to decide a simpler approximation formula of the numerical results. The obtained coefficients are shown in Table 6.

The numerical results are fitted better at high frequencies with $R^{2}$ parameter closer to unity. This can also be concluded by means of the standard error of the linear regression which is larger at low frequencies. It means that differences between the numerical results and the regression formula are larger due to the modal behaviour and the influence of the specific dimensions of the junction. The opposite can be said at high frequencies.

In general, more variables are required to describe the low-frequency zone. The influence of the frequency in the vibration reduction index trend is more important at the mid-frequency zone in the cases of straight transmission ( $\mathrm{T}$ and + -shaped, path 13 ). To include damping in the regression formula is more relevant at high-frequencies. An increase of the vibration reduction index at the high-frequency zone is observed in all $\mathrm{T}$ and +-shaped junctions analysed. An example of the improvement of the regression due to the consideration of damping is that the $R^{2}$ parameter varies from 0.68 to 0.83 in the right-angle transmission (path 1-2) of a + -shaped junction at high frequencies.

Considering the mass ratio $(M)$ is relevant in all junction types. In the right-angle transmission, as it happens in the EN-12354 formulas of Table 4 for the L and +shaped junctions, only $M^{2}$ (or $|M|$ ) is considered in the final formula. The significance of $M$ is very low and the regression analysis suggests to drop this variable. It is very logical due to symmetry reasons. However, this is not the case for the right-angle transmission in the T-shaped junction. EN-12354 formula keeps symmetry in this case and only depends on $M^{2}$ (not $M$ ). But the regression analysis of the numerical results suggests that $M$ is quite meaningful and improves the description of data. This makes sense because right-angle transmission in a T-shaped junction has no symmetry.

The formula Eq. (22) represents a simplified but good approximation of the numerical results. The variation of the vibration reduction index values is also caused by aspects different from the mass ratio, the frequency or the damping. Eq. (22) does not take into account the specific dimensions of the problem, the boundary conditions or the type and position of forces. 


\begin{tabular}{lllrrrrrrr} 
Junction & Path & Range & $\mathrm{a}$ & $\mathrm{b}$ & $\mathrm{c}$ & $\mathrm{d}$ & $\mathrm{e}$ & $R^{2}$ & Std. Err. \\
\hline T-shaped & $1-2$ & Low & -10.66 & -9.98 & 3.98 & 30.03 & 27.15 & 0.6 & 3.90 \\
T-shaped & $1-2$ & Mid & - & - & 4.89 & 28.95 & 5.24 & 0.81 & 2.04 \\
T-shaped & $1-2$ & High & 16.32 & - & 6.71 & 23.46 & 4.43 & 0.86 & 1.59 \\
\hline T-shaped & $1-3$ & Low & - & - & -18.58 & 33.82 & 7.32 & 0.86 & 3.98 \\
T-shaped & $1-3$ & Mid & - & -12.92 & -14.90 & 9.76 & 39.26 & 0.82 & 3.36 \\
T-shaped & $1-3$ & High & 18.99 & - & -10.30 & 7.65 & -2.52 & 0.91 & 1.49 \\
\hline +-shaped & $1-2$ & Low & -10.63 & -9.56 & -0.33 & 27.29 & 29.13 & 0.57 & 4.01 \\
+-shaped & $1-2$ & Mid & - & - & - & 25.96 & 7.11 & 0.78 & 2.11 \\
+-shaped & $1-2$ & High & 18.62 & - & - & 24.16 & 5.14 & 0.83 & 1.84 \\
\hline +-shaped & $1-3$ & Low & - & - & -24.94 & 31.03 & 9.51 & 0.89 & 4.25 \\
+-shaped & $1-3$ & Mid & - & -10.92 & -18.87 & 11.21 & 37.40 & 0.86 & 3.38 \\
+-shaped & $1-3$ & High & 19.24 & - & -13.83 & 6.65 & 0.58 & 0.93 & 1.70 \\
\hline L-shaped & $1-2$ & Low & -12.52 & -7.91 & -1.97 & 32.94 & 20.66 & 0.71 & 3.56 \\
L-shaped & $1-2$ & Mid & - & - & - & 23.67 & 3.78 & 0.70 & 2.58 \\
L-shaped & $1-2$ & High & 13.28 & - & - & 16.94 & 3.04 & 0.82 & 1.35 \\
\hline narrowing & $1-3$ & Low & -15.43 & -5.21 & - & 8.93 & 9.37 & 0.51 & 2.14 \\
narrowing & $1-3$ & Mid & - & - & - & 9.54 & -4.67 & 0.52 & 2.12 \\
narrowing & $1-3$ & High & 13.16 & - & 2.2 & 8.57 & -5.94 & 0.67 & 1.28 \\
\hline
\end{tabular}

Table 6: Regression coefficients. $M=\log _{10}\left(t_{1} / t_{2}\right)$ with $t_{1}=t_{3}$ in all cases except the narrowing where $M=\log _{10}\left(t_{2} / t_{1}\right)$.

\subsubsection{Single-parameter output}

A comparison with EN-12354 has also been done in terms of output averaged in the mid-frequency range. The EN-12354 propose a single value for the vibration reduction index which is frequency-independent.

Fig. 8 shows the values of $K_{13}$ (straight transmission) and Fig. 9 the values of $K_{12}$ (right-angle transmission) for a T-shaped junction. The parameter that varies here is the mass ratio because the thicknesses of plates forming the junction is not the same in each branch. In both Figs. 8 and 9 the influence of damping is shown in part (a) and the influence of the frequency range (low, mid or high) used to obtain $\overline{K_{i j}}$ is shown in the part (b).

A first thing to be noted in Figs. 8 and 9 (a) is that the vibration reduction curves for the different values of damping considered are very close. Differences in the midfrequency averaged vibration reduction index $\overline{K_{i j}}$ are not larger than $4 \mathrm{~dB}$ while as shown in Figs. 6 and 7 they can be larger than $10 \mathrm{~dB}$ for the vibration level difference $\overline{D_{\nu, i j}}$. It means that the definition of Eq. (3) is quite correct in the sense that makes the parameter more independent of damping. This is observed for all transmission paths $(1-2$ and $1-3)$. However, as mentioned before, this is not true for the poorly damped case $\eta=1 \%$ where differences in $\overline{K_{i j}}$ are more significant.

Figs. 8 and 9 (b) show the importance of the frequency range used in order to obtain $\overline{K_{i j}}$. Larger values of $\overline{K_{i j}}$ are obtained in the low-frequency average while $\overline{K_{i j}}$ is smaller in the high-frequency average. 
The agreement of the numerical (mid-frequency average) results with the EN12354 formulas of Table 4 is quite good for the straight transmission (path $1-3$, Fig. 8 (a)). Except for the $\eta=1 \%$ curves, differences are less or equal $\pm 3 \mathrm{~dB}$. It must be noted that these differences can also be found for cases with equal mass ratio $(M)$ value for which EN-12354 formulas propose the same vibration reduction index (i.e. $t_{1}=0.1, t_{2}=0.2$ and $t_{1}=0.2, t_{2}=0.4$ ). It makes sense because the different thicknesses imply different modal distributions and balances between in-plane and out-of-plane modes. To have the same mass ratio and the same plate dimensions does not imply that the junctions are identical and thus, small differences can be expected.

Similar comments are valid for the agreement in the right-angle transmission case of Fig. 8 (a) but only in the mass ratio range between -0.5 and $0.5\left(m_{\perp} / m_{i}\right.$ between 0.3 and 3). For junctions with branches having a very different thickness the differences between the numerical results and EN-12354 are important and can be around 6 and $10 \mathrm{~dB}$. In almost all cases the values proposed by EN-12354 are smaller.

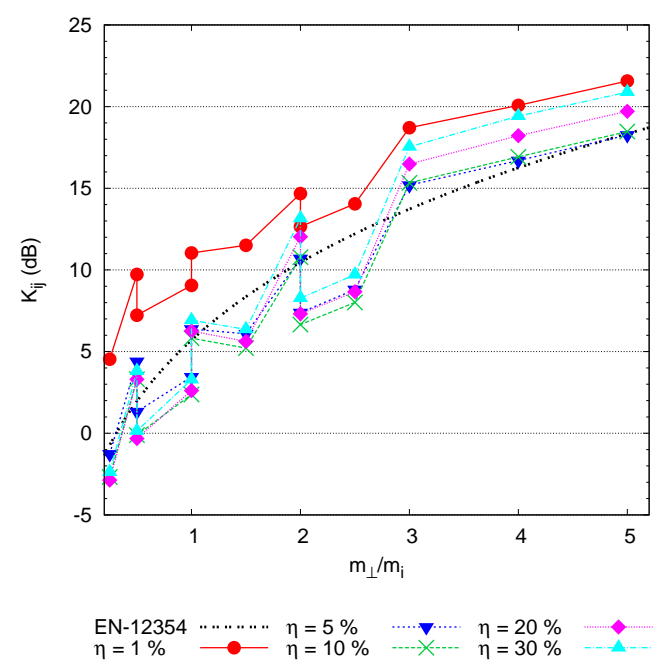

(a)

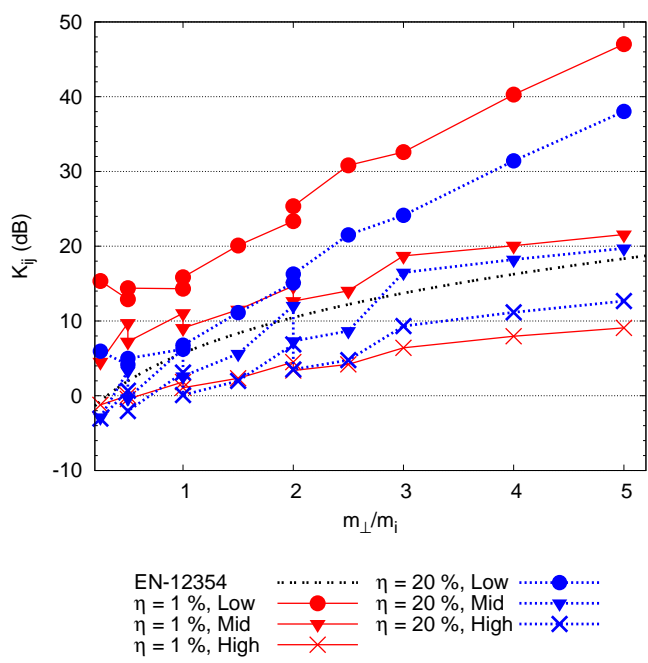

(b)

Figure 8: T-shaped junction, straight transmission $\left(\overline{K_{13}}\right)$ : (a) influence of damping (mid-frequency range average); (b) $\overline{K_{13}}$ averaged in the low, mid and high-frequency ranges.

An important aspect of the numerical model used here is that the finite dimensions of the junction can be considered. The results shown in previous pictures are obtained as the average of all the junctions described in Table 5 which is an ensemble of common building dimensions. It is, however, interesting to see how the dimensions of the junctions affect the standard deviation of the vibration reduction index. A first indicator is the standard error of the regressions formulas (see Table 6). The standard deviation of $\overline{K_{i, j}}$ for the T-shaped junction is shown in Fig. 10. The standard deviation decreases when damping or frequency increases. The largest standard deviation is found considering the low-frequency average and the case $\eta=1 \%$. Except for this extreme situation, it can be said that the standard deviation due to the plate dimensions is not larger than $4 \mathrm{~dB}( \pm 3 \mathrm{~dB}$ is mentioned in the ISO10848-1). The standard 


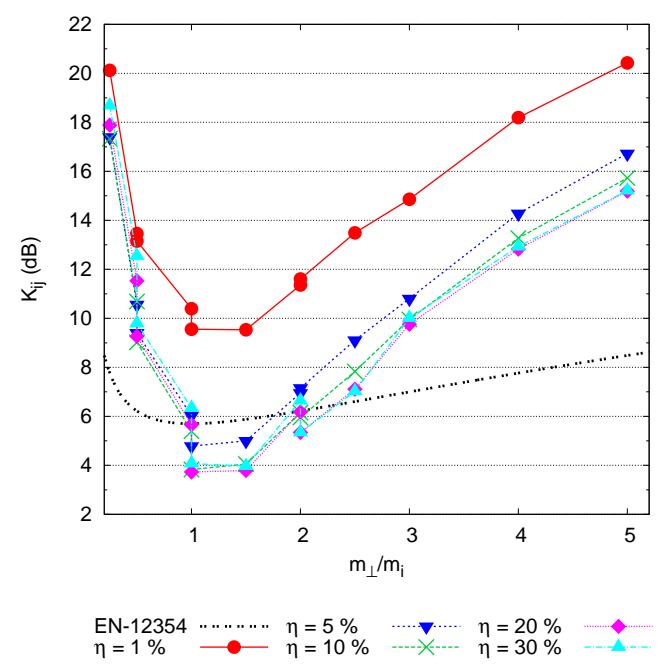

(a)
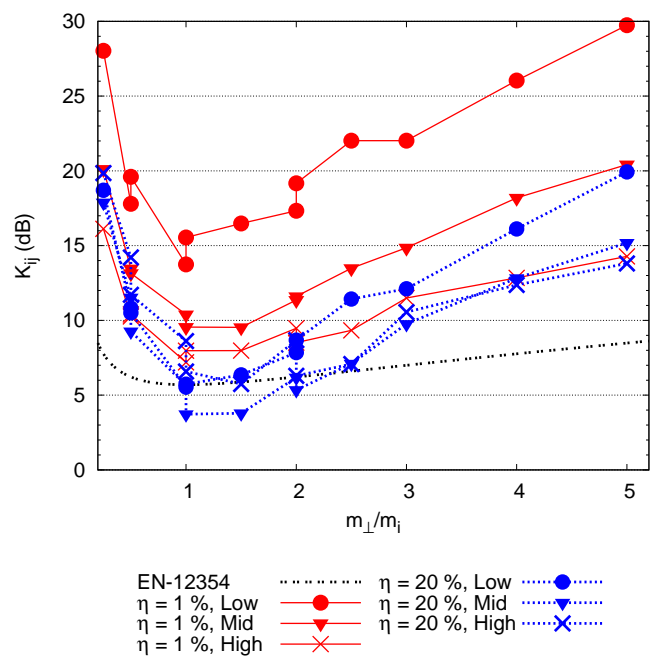

(b)

Figure 9: T-shaped junction, right-angle transmission $\left(\overline{K_{12}}\right)$ : (a) influence of damping (mid-frequency range average); (b) $\overline{K_{12}}$ averaged in the low, mid and high-frequency ranges.

deviation does not depend on the mass ratio.

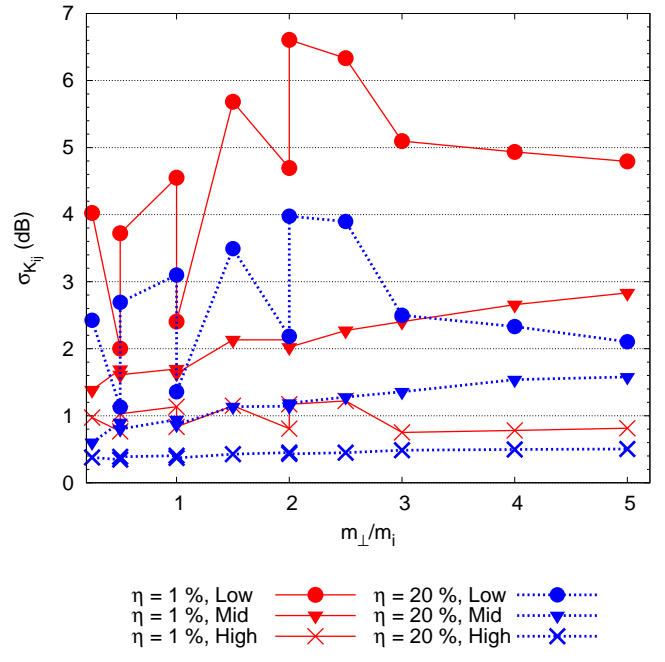

(a)

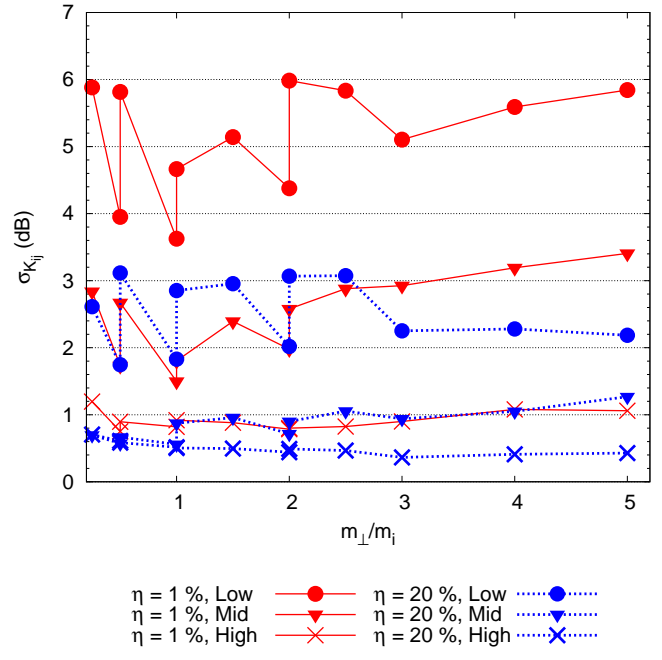

(b)

Figure 10: T-shaped junction, mean standard deviation averaged in the mid-frequency range (variation due to problem geometry): (a) straight transmission $\left(\overline{K_{13}}\right)$; (b) rightangle transmission $\left(\overline{K_{12}}\right)$.

A similar analysis has been done for the +-shaped (Fig. 11), L-shaped (Fig. 12(a)) and the sudden narrowing in a straight structure (Fig. 12(b)). The results obtained go in the same line as for the T-shaped. The most significant difference must be 
mentioned for the L-shaped where numerical results are systematically $5 \mathrm{~dB}$ above the EN-12354 formula.

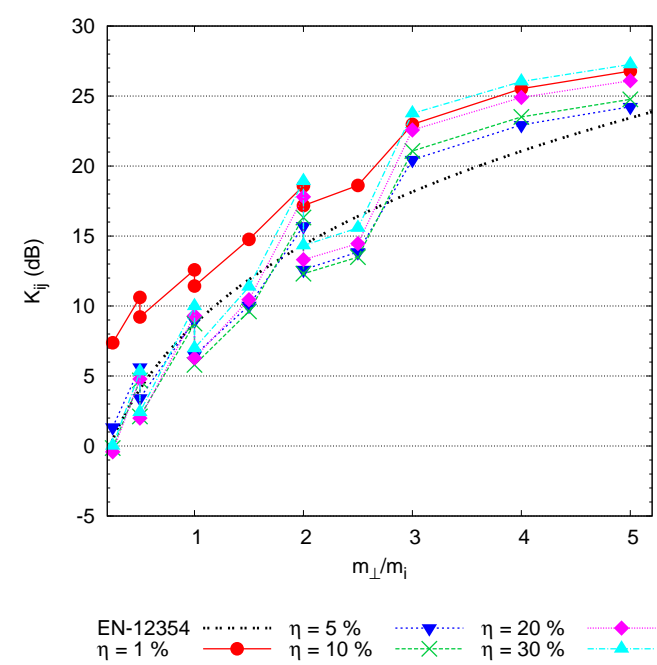

(a)

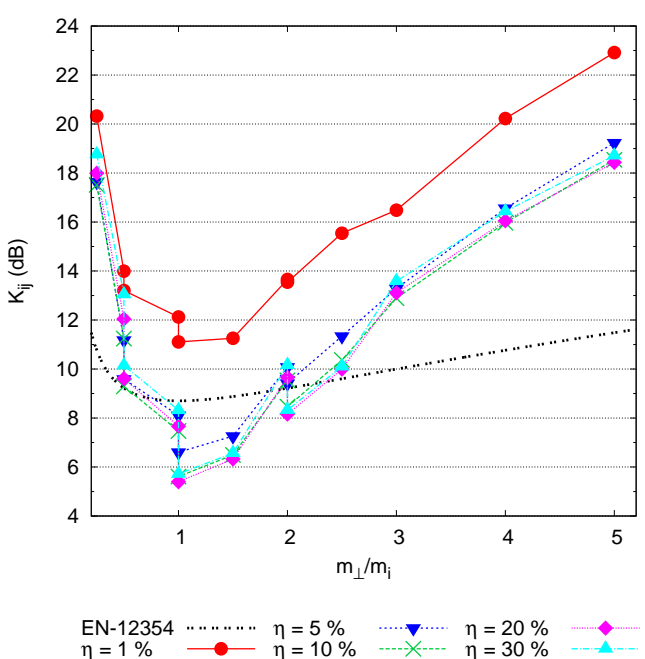

(b)

Figure 11: Vibration reduction index of the +-shaped junction, influence of damping averaging in the mid-frequency range: (a) straight transmission $\left(\overline{K_{13}}\right)$; (b) right-angle transmission $\overline{K_{12}}$.

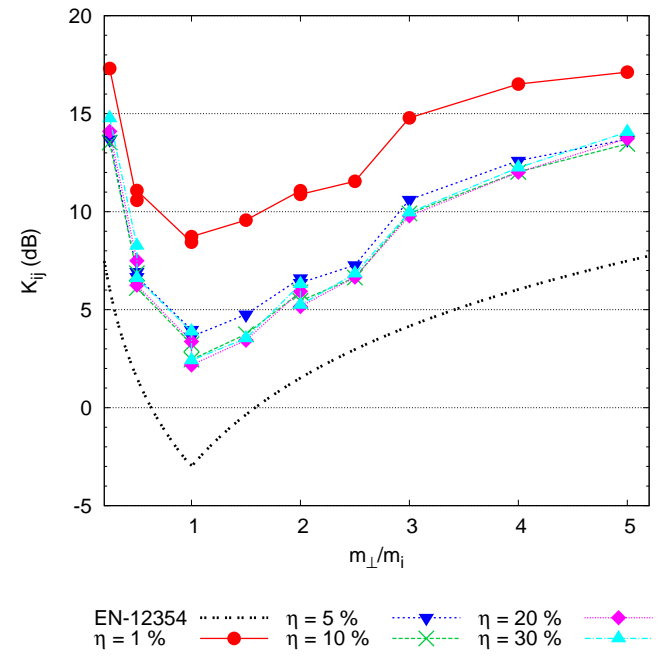

(a)
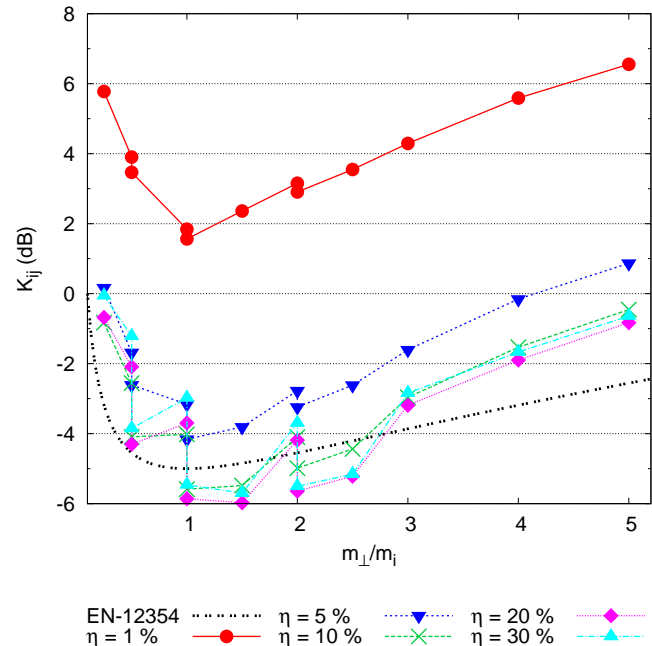

(b)

Figure 12: Vibration reduction index averaging in the mid-frequency range: (a)Lshaped junction, transmission $\left(\overline{K_{12}}\right)$; (b) transmission through a sudden narrowing in a straight structure $\left(\overline{K_{13}}\right)$.

Another aspect that can be analysed with the presented model is the relative importance of the out-of plane displacements with respect to the total displacement. 
Sometimes only bending waves are considered in the study of the transmission coefficient at junctions. The main reason is that equations become simple and it is easier (possible) to obtain analytical expressions for the transmission loss (see for example some of the models developed in [19, 24]), or the interest is only focused on the vibration transmission due to bending waves. However, it is well known that in-plane vibration is important at high frequencies and for thick structures. Some models of junctions including the effect of in-plane vibrations have also been developed [44, 19, 22, 45, 37].

This aspect is studied here in the case of the T-shaped junction with the excitation in the zone 1. The parameter shown in Fig. 13 is the ratio between the spatial averaged kinetic energy taking into account only the out-of-plane displacements and the same but taking into account also the in-plane displacements. The spatial average is done over the zone 2 of the T-shaped junction. When the ratio value is close to 1 , the inplane energy can be neglected. In the case where the point forces are applied directly in zone 2 this ratio is very close to 1 in all analysed cases.

Fig. 13 shows how in-plane effects are more important when the frequency increases. But they become meaningful already in the mid-frequency range. They are more sensitive to the thickness ratio (a) than to damping (b). When one of the plates becomes thicker, in-plane effects are more important. The effect of damping is different for low and high frequencies. According to these results, it can be very inaccurate to use models that take into account only bending waves in order to calculate the transmission coefficient of junctions where interaction between bending and in-plane waves of different elements can exist (i.e. right-angle transmission).

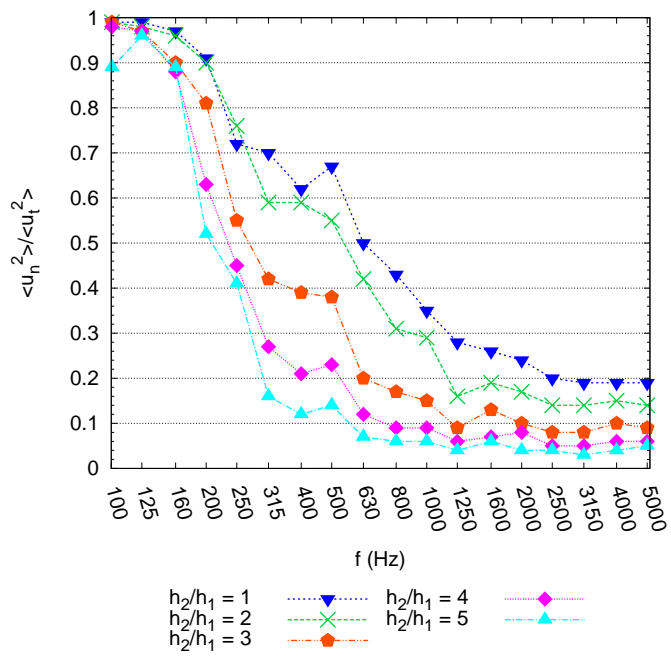

(a)

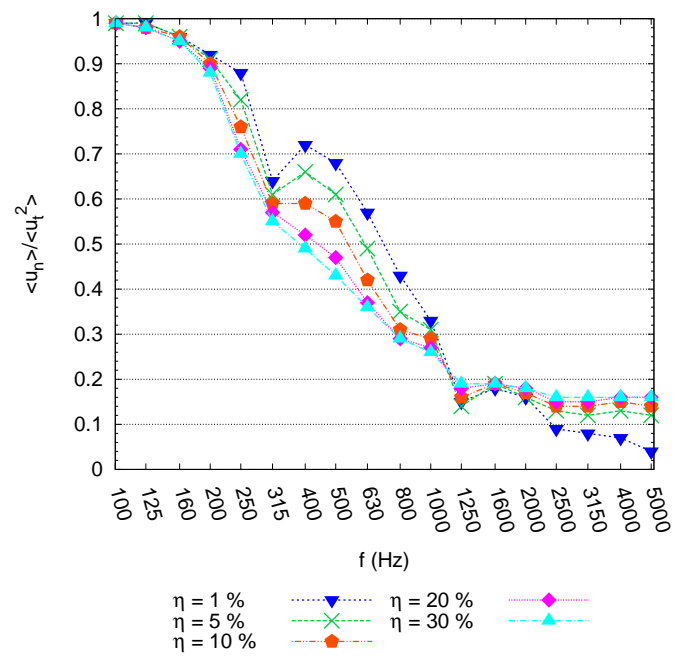

(b)

Figure 13: T-shaped junction, ratio of kinetic energy caused by out-of-plane displacement in zone 2 when the excitation is placed in the zone 1: (a) influence of thickness ratio $(\eta=10 \%)$; (b) influence of damping $\left(t_{1,3}=0.1 \mathrm{~m}\right.$ and $\left.t_{2}=0.2 \mathrm{~m}\right)$. 


\subsection{Effect of material stiffness}

EN-12354 formulas neglect the effect on the vibration reduction index of the Young modulus or the presence of branches with different stiffness. These two situations have been considered in a T-shaped junction of $0.1 \mathrm{~m}$ thick plates. The analysis shown in Figs. 8 and 9 is repeated by introducing two modifications:

- Variation of the Young modulus in the whole junction

- Variation of the Young modulus only in the vertical plate (number 2 according the notation of Fig. 5)

In both cases, the value of $E=3.0 \cdot 10^{10} \mathrm{~N} / \mathrm{m}^{2}$ is taken as reference. It is multiplied by: $1 / 10,1 / 2,1,2$ or 5 in the whole junction or only in the plate number 2 . Real construction materials rarely change their Young modulus without a change in density. However, we consider that it is a clear way to study the effects of the material stiffness variation in the value of the vibration reduction index. Damping is constant in this section with $\eta=10 \%$.

The variation of the Young modulus of the vertical plate (number 2) cause important variations on the vibration reduction index of the T-shaped junction. On the one hand the straight transmission (path 1-3 see Fig. 15(a)) decreases for a stiffer vertical plate. On the other hand, the right-angle transmission (path $1-2$ see Fig. 14(a)) decreases for a stiffer vertical plate if this plate number 2 is thicker than the others $(1$ and 3). If plate number 2 is thinner than the others, the effect of a Young modulus increase is inverse and cause an increase on the vibration transmission. Note that, as in previous sections, talking about 'mass ratio' or 'thickness ratio' is equivalent due to the constant value of volume density.

When the Young modulus is changed in the whole junction, the variations of vibration reduction index are less important but still significant. In the straight transmission (path $1-3$ see Fig. 15(b)) the variations of $\overline{K_{13}}$ are larger than $4 \mathrm{~dB}$ only for junctions with $m_{\perp} / m_{i}$ larger than 2. On the contrary, for the right-angle transmission (path $1-2$ see Fig. 14(b)) the larger variations of $\overline{K_{12}}$ are found for $m_{\perp} / m_{i}$ smaller than 2 .

In any of the cases, the stiffness of the material is an important parameter that can affect the values of vibration reduction index in one sense or another. Similar conclusions have been drawn for the other junction types considered in the analysis.

\subsection{Effect of the surrounding building structures}

Other aspects not related with the junction but with the boundary conditions can have certain influence on the vibration reduction index results too. They cannot be considered in the EN-12354 simplified equations and in most of the junction models based on wave-approach [19, 24].

A first aspect is the excitation force. Fig. 16 show a comparison of the vibration reduction index obtained with the three point forces commented before or single point forces. The single forces are placed at the corner (at a distance of $1.0 \mathrm{~m}$ of the junction and the border of the plate) or at the centre of the excited plate. The differences caused by the single point force position are around $3-5 \mathrm{~dB}$ in all the frequency 


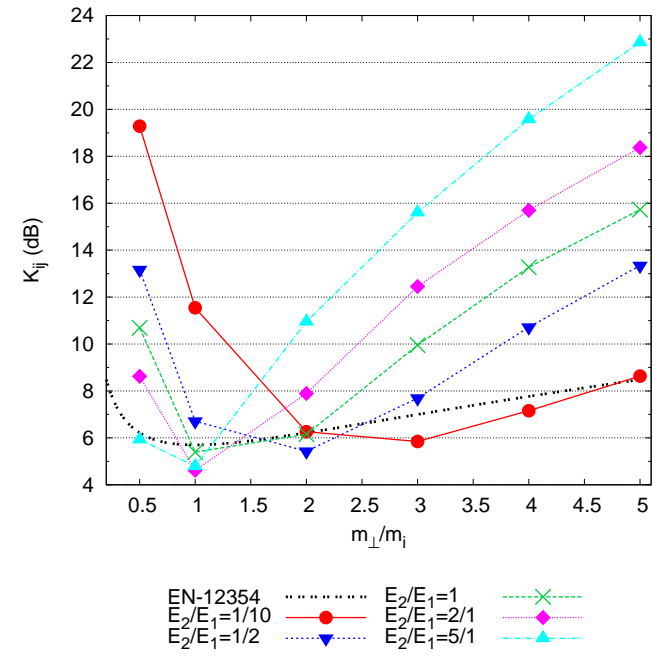

(a)

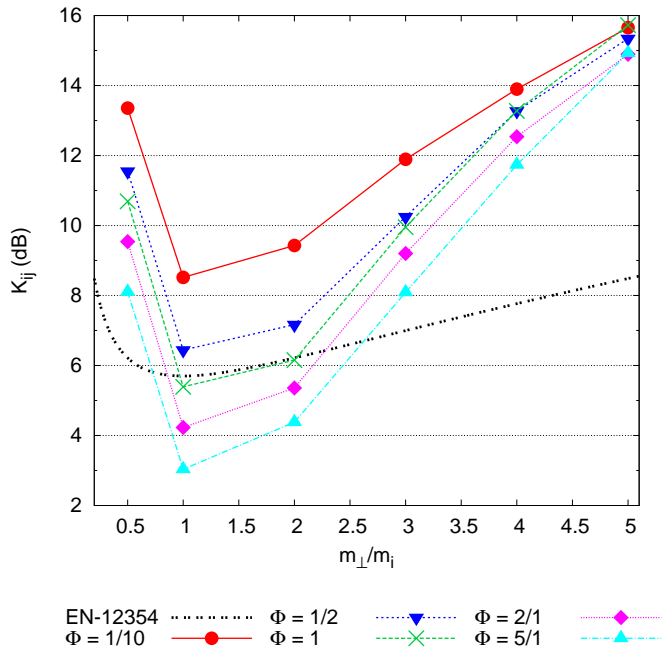

(b)

Figure 14: T-shaped junction, right-angle transmission $\overline{K_{12}}$, influence of Young modulus ( $\eta=10 \%, \overline{K_{12}}$ averaged in the mid-frequency range): (a) The Young modulus of plates 1 and 3 is constant $\left(E_{1}=E_{3}=30 \mathrm{GPa}\right)$ and the Young modulus of plate 2 $\left(E_{2}\right)$ is variable; (b) Same Young modulus $E_{\text {all }}$ in the whole junction, $\Phi=E_{\text {all }} / E_{\text {ref }}$ with reference value $E_{\text {ref }}=30 \mathrm{GPa}$.

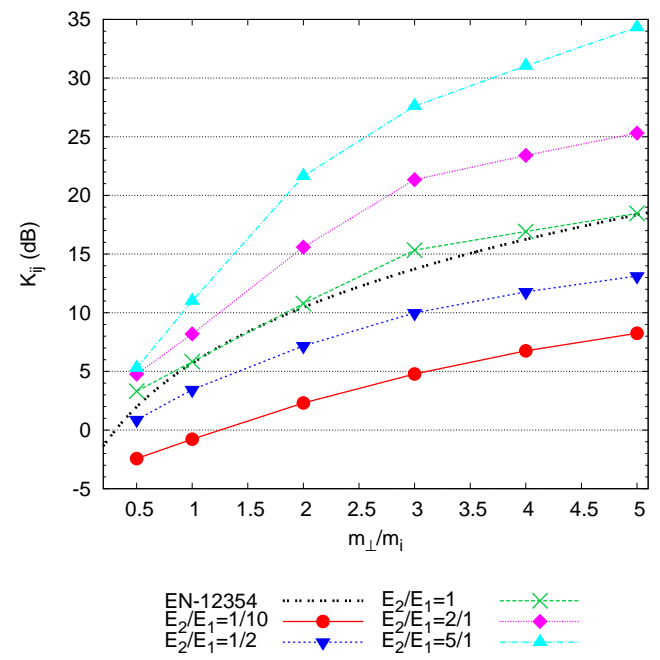

(a)

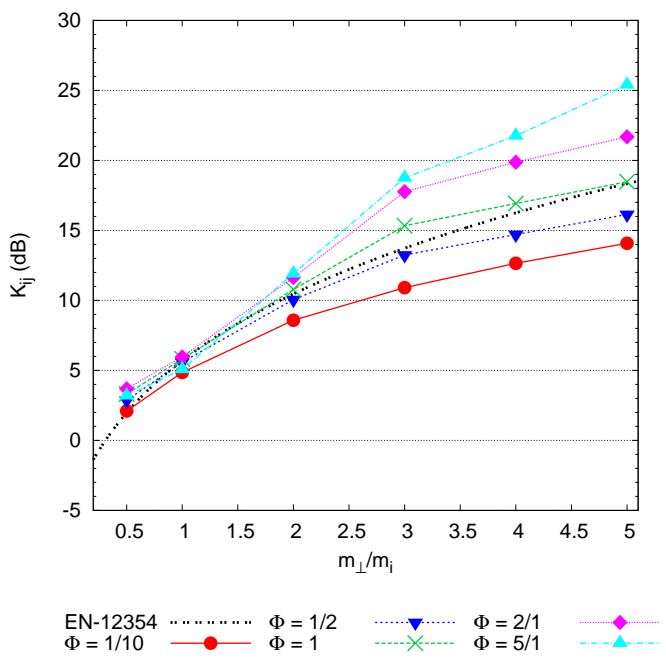

(b)

Figure 15: T-shaped junction, straight transmission $\overline{K_{13}}$, influence of Young modulus ( $\eta=10 \%, \overline{K_{13}}$ averaged in the mid-frequency range): (a) The Young modulus of plates 1 and 3 is constant $\left(E_{1}=E_{3}=30 \mathrm{GPa}\right)$ and the Young modulus of plate 2 $\left(E_{2}\right)$ is variable; (b) Same Young modulus $E_{\text {all }}$ in the whole junction, $\Phi=E_{\text {all }} / E_{\text {ref }}$ with reference value $E_{\text {ref }}=30 \mathrm{GPa}$. 
range with a damping $\eta=30 \%$. These differences decrease if damping is reduced and are less than $2 \mathrm{~dB}$ for the case $\eta=10 \%$. The curve with three point forces applied at the same time is, as expected, between the other two cases.

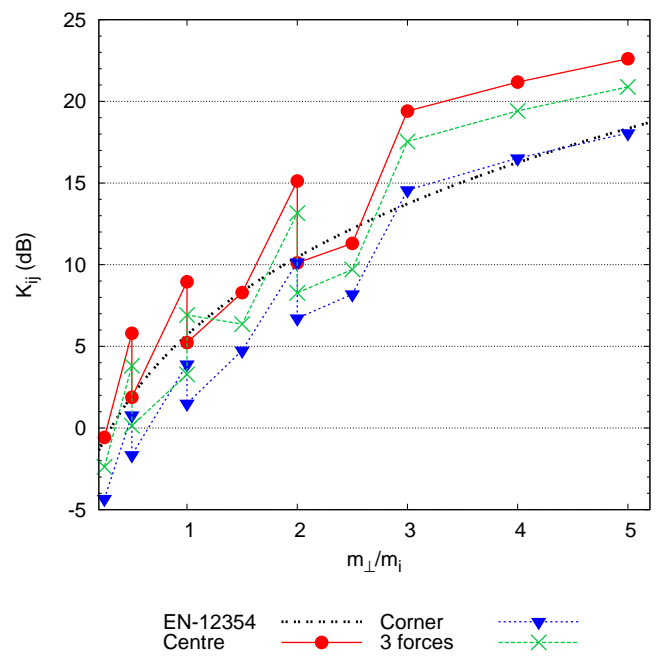

(a)

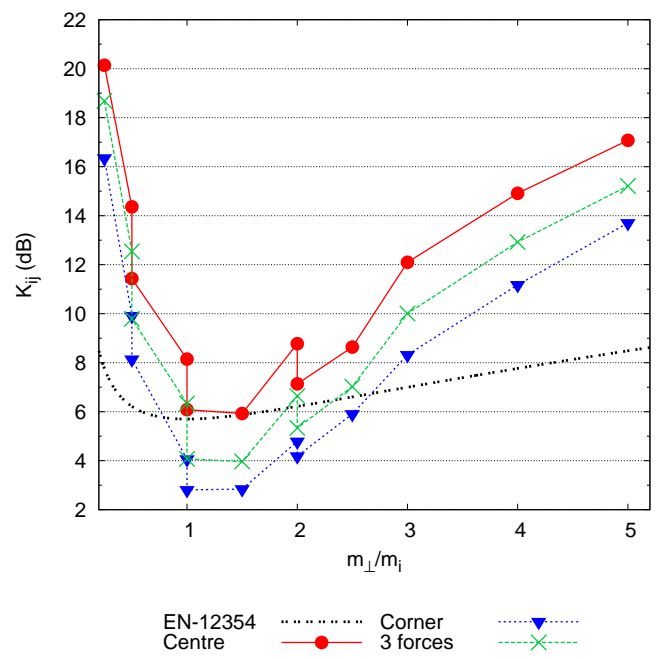

(b)

Figure 16: T-shaped junction ( $\eta=30 \%, \overline{K_{i j}}$ averaged in the mid-frequency range), influence of the force position on the vibration reduction index: (a) straight transmission $\overline{K_{13}}$; (b) right-angle $\overline{K_{12}}$.

The generality of the numerical tool can be used in order to analyse more complex structures. The differences in the vibration reduction index of an isolated +-shaped junction (results presented in Fig. 11 ) and the same +-shaped junction placed inside the framework of a building structure (see Fig. 17(b)) has been calculated. All the junctions are rigid line connections in the building structure. Results are shown in Fig. 17(a) and reveal that differences are not large. It seems justified to study the junctions isolated from the other parts of the structure as EN-12354 formulas propose. However, it is a preliminary example that illustrates the possibilities offered by the model presented in Section 2 in order to study more complex situations (i.e. building frameworks). A larger study must be done in order to obtain general conclusions or design rules, in the line of previous works [46]. 


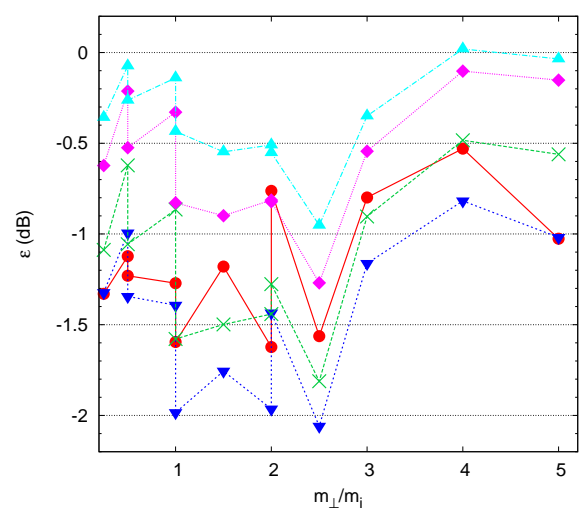

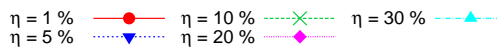

(a)

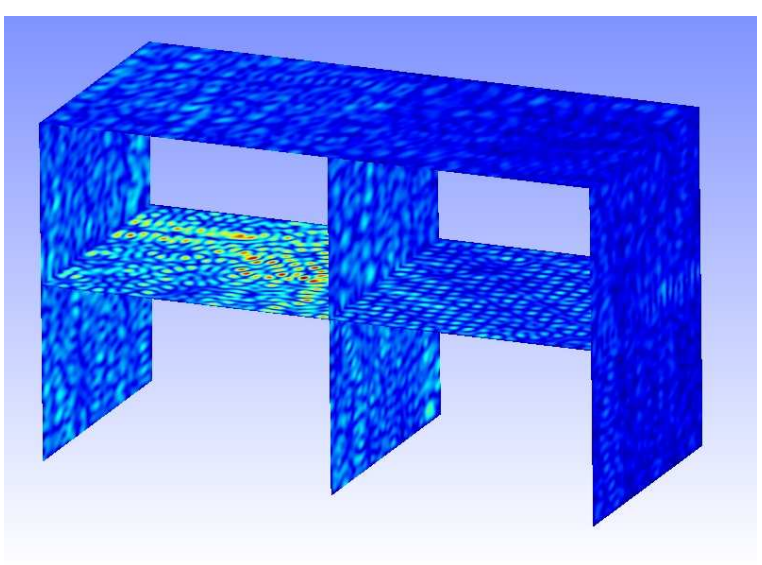

(b)

Figure 17: +-shaped junction ( $K_{i j}$ averaged in all the mid-frequency range), influence of the boundary conditions on the vibration reduction index: (a) differences in straight transmission $K_{13}$ between an isolated + -shaped junction and the same junction places inside a 'building structure': $\varepsilon=K_{i j}^{\text {isolated }}-K_{i j}^{\text {building }}$; (b) Absolute modulus of displacement at the 'building structure at $4000 \mathrm{~Hz}: \eta=1 \%$, all thicknesses of $0.1 \mathrm{~m}$. 


\section{Conclusions}

The main conclusions of the analysis are summarised here below:

- The implemented model, which is based on the spectral element method, provides results that are equivalent to three-dimensional FEM. The systems of equations to be solved are much smaller and consequently the results can be obtained in a much faster and efficient way. This has been the key in order to perform this broad analysis extended in the whole frequency range.

- The vibration reduction index is a parameter that exhibits less variation than the vibration level difference. However, the influence of the plate dimensions is still important and can be around $1.5 \mathrm{~dB}$ for highly damped structures or almost $3.5 \mathrm{~dB}$ for the poorly damped ones. It must be mentioned that the idea of an invariable vibration reduction index seems to be non valid when $\eta=1 \%$ or less.

- The vibration reduction index curves obtained with SFEM present a non-negligible frequency-dependence. This is more important in the straight transmission (path $1-3$ ) for the + and T-shaped junctions. The general trend is that $K_{i j}$ decreases with frequency $\left(\propto-10 \log _{10}(f)\right)$. In the low-frequency range, the calculated values are larger than EN-12354 predictions. In the high-frequency range and for the most damped structures $K_{i j}$ can have a moderate increase. Both aspects coincide with experimental measurements already reported in [25, 13].

- Difference with the EN-12354 prediction formulas are more important for junctions with larger mass ratio and for those cases of right-angle transmission (i.e. from floor to wall). In general, the EN-12354 formulas provide smaller values than the numerically calculated ones.

- Other parameters such as the Young modulus or the force position have importance in the prediction of the vibration reduction index. The largest differences with respect to EN-12354 prediction formulas are found when there are nonhomogeneous junctions (i.e. different Young modulus for perpendicular plates). Considering point forces in different positions makes a variation of results around $3 \mathrm{~dB}$ for highly damped junctions $(\eta=30 \%)$. However, very similar results have been obtained for the vibration reduction index of a + -shaped junction isolated or forming part of a building framework. The largest differences are found for the lower damping of $\eta=1 \%$ but are less than $2 \mathrm{~dB}$.

- It is very important, for the heavy junctions considered here, to take into account the in-plane stiffness and longitudinal waves. This is more relevant in the rightangle transmissions. In that cases and for mid and high frequencies, the out-ofplane displacement can be less that $25 \%$ of the total displacement. 


\section{Acknowledgements}

The authors appreciate very much the extra information and data received from Charlotte Crispin (Centre Scientifique et Technique de la Construction). Free software has been used [47, 39, 48].

\section{References}

[1] C. Hopkins. Sound transmission across a separating cavity wall construction and flanking. Appl. Acoust., 52(3-4):259-272, 1997.

[2] R.J.M. Craik. The contribution of long flanking paths to sound transmission in buildings. Appl. Acoust., 62:29-46, 2001.

[3] J.L. Guyader, C. Boisson, C. Lesueur, and P. Millot. Sound transmission by coupled structures: application to flanking transmission in buildings. J. Sound Vibr., 106(2):289-310, 1986.

[4] D. Clasen and S. Langer. Finite element approach for flanking transmission in building acoustics. J. Building Acoustics, 14(1):1-14, 2007.

[5] J.A. Steel and R.J.M. Craik. Statistical energy analysis of structure-borne sound transmission by finite element methods. J. Sound Vibr., 178(4):553-561, 1994.

[6] C. Hopkins. Vibration transmission between coupled plates using finite element methods and statistical energy analysis. Part 1: Comparison of measured and predicted data for masonry walls with and without apertures. Appl. Acoust., 64(10):955-973, 2003.

[7] E. Gerretsen. Calculation of the sound transmission between dwellings by partitions and flanking structures. Appl. Acoust., 12(6):413-433, 1979.

[8] E. Gerretsen. Calculation of airborne and impact sound insulation between dwellings. Appl. Acoust., 19(4):245-264, 1986.

[9] EN-12354. Building Acoustics: Estimation of the acoustic performance of buildings from the performance of elements. (Acoustique du bâtiment: Calcul de la performance acustique des bâtiments à partir de la performance des éléments). Technical Report 1-4, 1999-2000.

[10] J. Mahn and J. Pearse. On the uncertainty of the EN12354-1 estimate of the flanking sound reduction index due to the uncertainty of the input data. $J$. Building Acoustics, 16(3):199-231, 2009.

[11] J. Mahn. Prediction of flanking noise transmission in lightweight building constructions: A theoretical and experimental evaluation of the application of EN 12354-1. PhD thesis, University of Canterbury. Department of Mechanical Engineering, 2009. 
[12] E. Gerretsen. Vibration reduction index $\mathrm{K}_{i j}$, a new quantity for sound transmission at junctions of building elements. In International congress on noise control engineering, pages 1475-1480, 1996.

[13] Ch. Crispin, B. Ingelaere, M. Van Damme, and D. Wuyts. The vibration reduction index $\mathrm{K}_{i j}$ : Laboratory measurements for rigid junctions and for junctions with flexible interlayers. J. Building Acoustics, 13(2):99-112, 2006.

[14] Ch. Crispin, M. Mertens, B. Blasco, B. Ingelaere, M. Van Damme, and D. Wuyts. The vibration reduction index $\mathrm{K}_{i j}$ :laboratory measurements versus predictions EN 12354-1 (2000). In The 33rd international congress and exposition on noise control engineering, Prague, 2004.

[15] A. Schiavi and A. Astolfi. The prediction of the vibration reduction index $\mathrm{K}_{i j}$ for brick and concrete rigid junctions. Appl. Acoust., 71(6):523-530, 2010.

[16] C. Hopkins. Measurement of the vibration reduction index, $\mathrm{K}_{i j}$ on free-standing masonry wall constructions. J. Building Acoustics, 6(3-4):235-257, 1999.

[17] J. Ramis, E. Segovia, J. Alba, J. Carbajo, and L. Godinho. Numerical evaluation of the vibration reduction index for structural joints. Archives of acoustics, 37(2):189-197, 2012.

[18] C. Guigou-Carter, C. Coguenanff, S. Bailhache, and M. Villot. Modeling lightweight junctions. In InterNoise proceedings, Noise control for quality of life, Innsbruck, 2013.

[19] L. Cremer, M. Heckl, and B. Petersson. Structure-borne sound: structural vibrations and sound radiation at audio frequencies. Springer, 2005.

[20] V. J Stauskis and M. Mickaitis. Vibration attenuation at asymmetric cross-form joints of buildings. Journal of Civil Engineering and Management, 11(2):129-135, 2005.

[21] P. Mees and O. Vermeir. Structure-borne sound transmission at elastically connected plates. J. Sound Vibr., 166(1):55-76, 1993.

[22] M.D. McCollum and J.M. Cuschieri. Bending and in-plane wave transmission in thick connected plates using statistical energy analysis. J. Acoust. Soc. Am., 88(3):1480-1485, 1990.

[23] M.B. Skeen and N.J. Kessissoglou. An investigation of transmission coefficients for finite and semi-infinite coupled plate structures. J. Acoust. Soc. Am., 122:814, 2007.

[24] R.J.M. Craik. Sound transmission through buildings using statistical energy analysis. Gower, England, 1996.

[25] D.B. Pedersen. Estimation of vibration attenuation through junctions of building structures. Appl. Acoust., 46(3):285-305, 1995. 
[26] Carl Hopkins. Sound insulation. Routledge, 2012.

[27] J.L. Guyader, C. Boisson, and C. Lesueur. Energy transmission in finite coupled plates, part I: Theory. J. Sound Vibr., 81(1):81-92, 1982.

[28] C. Boisson, J.L. Guyader, P. Millot, and C. Lesueur. Energy transmission in finite coupled plates, part II: Application to an L shaped structure. J. Sound Vibr., 81(1):93-105, 1982.

[29] C. Simmons. Structure-borne sound transmission through plate junctions and estimates of SEA coupling loss factors using the finite element method. J. Sound Vibr., 144(2):215-227, 1991.

[30] Ph. Jean and J. Roland. Simplified SEA-type models to predict the sound insulation between dwellings. Appl. Acoust., 40(3):201-211, 1993.

[31] M. Villot. Modeling flanking transmissions in lightweight constructions. In Forum acusticum, Sevilla, 2002.

[32] J. Poblet-Puig, A. Rodríguez-Ferran, C. Guigou-Carter, and M. Villot. Numerical modelling of the radiation efficiency of asymmetrical structures. Appl. Acoust., 70(5):777-780, 2009.

[33] T.R.T. Nightingale. Application of the CEN draft building acoustics prediction model to a lightweight double leaf construction. Appl. Acoust., 46(3):265-284, 1995 .

[34] C. Guigou-Carter, M. Villot, and R. Wetta. Prediction method adapted to wood frame lightweight constructions. J. Building Acoustics, 13(3):173-188, 2006.

[35] J.L. Davy, J.P. Mahn, C. Guigou-Carter, and M. Villot. The prediction of flanking sound transmission below the critical frequency. J. Acoust. Soc. Am., 132:23-59, 2012 .

[36] J.F. Doyle. Wave propagation in structures: spectral analysis using fast discrete fourier transforms. Springer, New York, 1997.

[37] N.J. Kessissoglou. Power transmission in L-shaped plates including flexural and in-plane vibration. J. Acoust. Soc. Am., 115:1157, 2004.

[38] Sh. H. Hashemi and A.R. Moradi. Exact solutions for the in-plane vibrations of rectangular Mindlin plates using Helmholtz decomposition. Acta mechanica, 215(1-4):345-361, 2010.

[39] EDF. Code-Aster home page. http://www.code-aster.org, 2014.

[40] Ch. Crispin. Private communication: Mesure de l'indice d'affaiblissement vibratoire, Kij, selon le projet de norme prEN 10848-1. Centre Scientifique et Technique de la Construction, 2014. 
[41] ISO. 10848-1:2006 acoustics - laboratory measurement of the flanking transmission of airborne and impact sound between adjoining rooms - part 1: Frame document. Technical report, International Organization for Standardization, 2006.

[42] S.F. Nabavi. Influence of polymers on concrete damping properties. In Proceedings of the European conference of chemical engineering, and European conference of civil engineering, and European conference of mechanical engineering, and European conference on Control, pages 28-33. World Scientific and Engineering Academy and Society (WSEAS), 2010.

[43] M. Villot and C. Guigou-Carter. Measurement methods adapted to wood frame lightweight constructions. J. Building Acoustics, 13(3):189-198, 2006.

[44] Tor Kihlman. Transmission of structure-borne sound in buildings: A theoretical and experimental investigation. National Swedish Inst. for Building Research, 1967.

[45] J.A. Moore. Vibration transmission through frame or beam junctions. J. Acoust. Soc. Am., 88(6):2766-2776, 1990.

[46] C. Hopkins and M. Robinson. On the evaluation of decay curves to determine structural reverberation times for building elements. Acta Acust. United Acust., 99(2):226-244, 2013.

[47] C. Geuzaine and J.-F. Remacle. Gmsh: a three-dimensional finite element mesh generator with built-in pre- and post-processing facilities. Int. J. Numer. Meth. Engng., 11(79):1309-1331, 2009.

[48] B. Pfaff, J. Darrington, et al. GNU PSPP home page. http://www.gnu.org/software/pspp/, 2014. 